bioRxiv preprint doi: https://doi.org/10.1101/2021.06.22.449446; this version posted September 9,2021 . The copyright holder for this preprint (which was not certified by peer review) is the author/funder, who has granted bioRxiv a license to display the preprint in perpetuity. It is made available under aCC-BY 4.0 International license.

\title{
Anti-clotting functions of lymphatics form the natural on-off switch for immune recognition by controlling the antigens and immune cells access to the lymph nodes
}

Witold W Kilarski ${ }^{1,2, \bigotimes, \Omega}$, Małgorzata Wachowska ${ }^{3}$, Angelika Muchowicz ${ }^{3}$, Renata Mezyk-Kopec ${ }^{4}$, Jakub Goląb ${ }^{3}$ \& Melody A Swartz ${ }^{1,2,5, \bigotimes, \Omega}$

The ability of lymph to clot indicates that similar to blood vessels lymphatics must have means to counteract this process. We analyzed their hemostatic properties, tailoring them for the potential therapeutic applications. Inflammatory stimuli induced tissue factor-dependent focal lymph clotting while blocking thrombomodulin lead to widespread but also transient occlusion of collecting vessels. Decellularization of lymphatics resulted in tissue factor-independent lymphatic occlusion by widespread and persistent lymph clots. These decellularized basement membrane remnants of collectors were capable of macromolecules drainage and leukocytes transit only when lymph clotting was inhibited with heparin. In occluded 'ghost' vessels, fibrin was replaced with transient basement membrane-rich inclusions to be eventually reperfused. During regeneration, ghost vessels were filled with granuloma-like clusters of antigen-presenting and $T$ cells. Despite that, immune response against allografts placed under non-drained skin was not developed as long lymphatics remained occluded, and graft survival was prolonged together with the delay of lymphatic regeneration with anti-lymphangiogenic therapy. Other potential applications of lymphatic hemostatic control functions include blocking pathogen or metastasis spread but also should be considered in the treatment of lymphedema.

Intima lining of blood vasculature forms the only known surface that is able to control blood liquidity ${ }^{1}$ dynamically, and despite ontogenetic and functional similarities, that function has not been yet ascribed to its relative circulation of lymphatic vessels. However, since the first description of the lymphatic system by Olaf Rudbeck over 350 years ago, lymph is known for its ability to coagulate when stored outside of the lymphatic vessels (discussed $\left.i^{2,3,4}\right)$. Interestingly, the above references illustrate that most of the research on lymph clotting was conducted over 50 years ago with little impact on the understanding of lymphatic related pathologies or therapeutic application ${ }^{5}$. Indeed, even though lymph clotting time is delayed as compared to blood, it has intrinsic clotting properties ${ }^{6,78}$. Its clotting time varies in a broad, up to 20-times range as a concentration of lymph clotting factors that fluctuate between species, individuals or even sampling location, and is 10 to $70 \%$ lower as compared to the blood ${ }^{9-11}$. Together with the absence of platelet-derived anionic phospholipids required for activation of Xase and prothrombinase and a higher concentration of fibrinolytic factors assure lymph hypercoagulability under physiological conditions ${ }^{8,}{ }^{12}$. Also, due to the lack of thrombocytes, lymph, in contrast to blood clots, do not retract ${ }^{8}$. On the other hand, relatively slow lymph flow and possibility to substitute platelet phospholipids with membrane components of endothelial cells ${ }^{13}$ may favor lymph clotting for example, during inflammation, when augmented blood vessel permeability to plasma proteins leads to 3 -fold enrichment of plasma proteins in interstitial fluid and lymph ${ }^{14}$. While data are missing for the inflammation or injury-driven concentration change of individual clotting factors in the lymph, it is rather safe to assume that concentration of coagulation components would rise when the rate of protein extravasation from blood vessels increases up to 100 times normalvcv $2,15,16$.

These observations confirm that lymph is a hemostatic fluid, but more importantly, it infers that similar to blood vessels, lymphatics must have specific anti-coagulatory properties that enable them to control lymph fluidity. By doing so, the lymphatic system could regulate other passive functions of lymphatics, i.e., fluid drainage and cell trafficking, and similar to blood vessels use pro-hemostatic mechanisms to limit the spread of infections ${ }^{17}$. However, only a handful of studies address thrombo-regulatory properties of lymphatic endothelium (for review see ${ }^{12}$ ). For example, it was shown that similar to blood endothelial cells; lymphatic collectors express thrombomodulin, a membrane receptor capable of switching the substrate specificity of thrombin to protein $\mathrm{C}$, thus making thrombin a potent anticlotting factor ${ }^{18}$. On the other hand, lymphatic endothelium does not produce von Willebrand factor, a multimeric protein that participates in platelet activation on the exposed subendothelial collagen. This phenotype, however, is in line with the lack of platelets in lymph ${ }^{19}$.

In response to pro-inflammatory molecules such as cytokines TNF $\alpha$, IL-1 $\beta$ or TLR agonist LPS, blood endothelium can turn into a pro-thrombotic surface, e.g., by initiating the expression of tissue factor (TF), membrane receptor initiating extrinsic coagulation pathway $^{20}$. Few in vitro studies have demonstrated that lymphatic endothelial cells are also capable of responding to inflammatory stimuli, for example with increased synthesis of plasminogen activators and inhibitors ${ }^{12,21}$. 


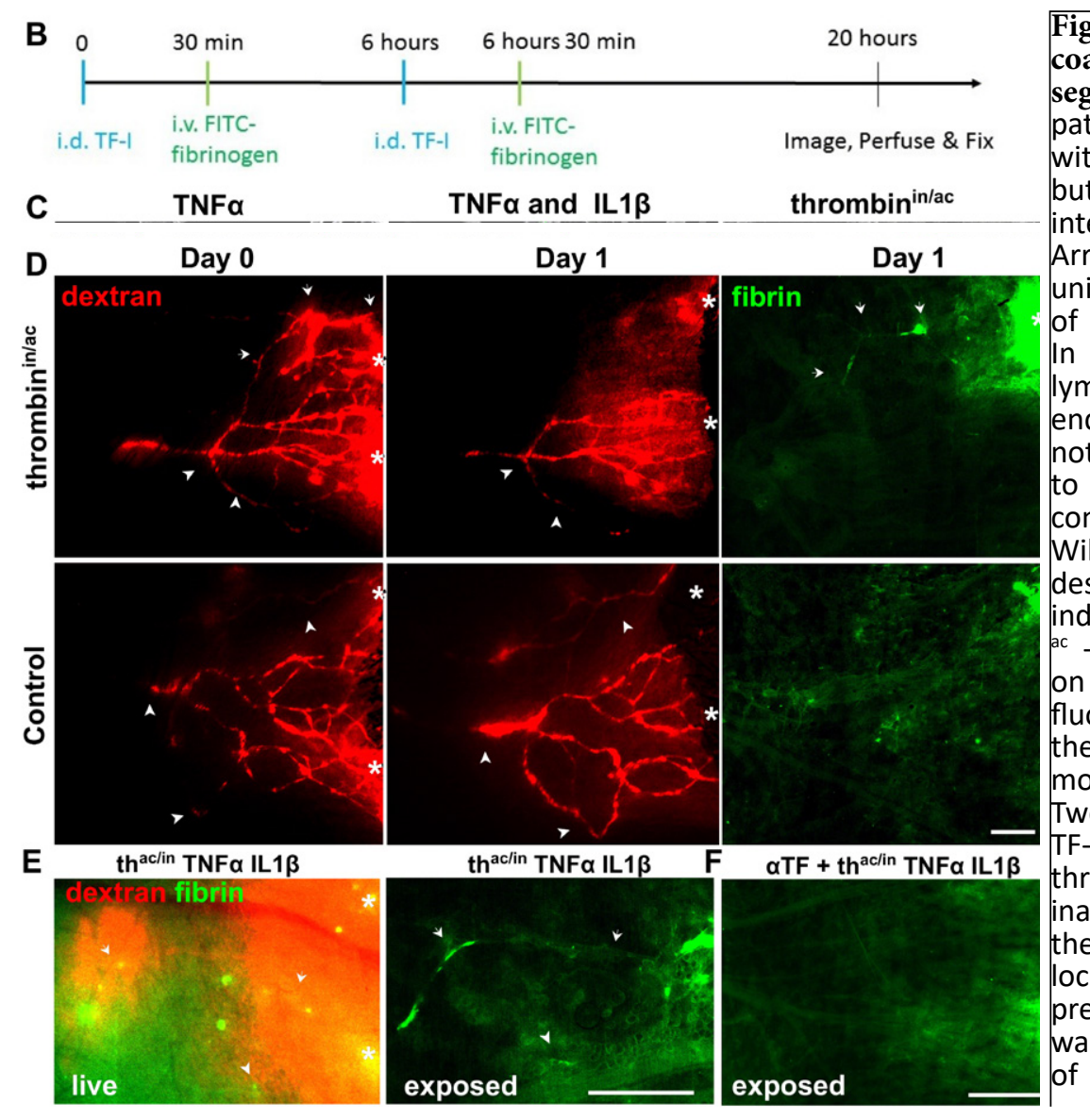

coase 1. Inflammatory mediators lead to lymph egments of lymphatic collectors. A. Left: The pattern of thrombomodulin expression overlapped
with the expression of endothelial marker CD31 but was also present on some perivascular and
interstitial cells but also within nerve fibers (n). Arrows point to collecting lymphatics that have Unique morphological features, i.e., the presence
of valves with uneven vessel diameters. Middle In non-inflamed ear skin, endothelial cells of
ymphatic collectors (LCES) similar to lo bloo
endothelium (BECS) express thrombomodulin but ndothelium (BECS) express thrombomodulin bu not tissue factor (TF), whose expression is restricted
o perivascular cells and adipocytes (a). Right: In of perivascular cells and adipocytes (a). Right:
contrast to BECS, LECS did not accumulate the von
Willebrand factor. B Schematic of experimental design. Intradermal injection of tissue factor nducers (TF-I TNF $\alpha$, TNF $\alpha$, and ILI $\beta$ or thrombin
- partially inactivated thrombin, see methods on day 0 was followed by intravenous injection of he first injection the procedure is repented. The mouse is perfused and fixed 13 hours later. $\mathrm{C}$

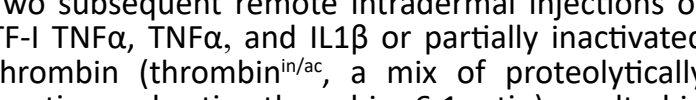
nactive and active thrombinin; $6: 1$ ratiol resulted in
ne formation of intralymphatic clots within specifit ocations of lymphatic collectors as shown by the presence of fluorescent fibrin. Basement membrane
was stained by collagen IV. D. Top: Demonstration vol Iymphatic drainage by intradermal injection of
of

fluorescent dextran. Two consecutive injections (6 hours apart on day 1 ) of thrombinin/ac blocked lymphatic drainage in some
branches of the collecting lymphatic network 12 hours after the second injection. Intralymphatic clots (fibrin) are present in with branches of the collecting lymphatic network 12 hours after the second injection. Intralymphatic clots (fibrin) are present in with
the location of Iymphatic collector junction functional lymphatic was blocked (arrows). Bottom: Intradermal injections of PBS had

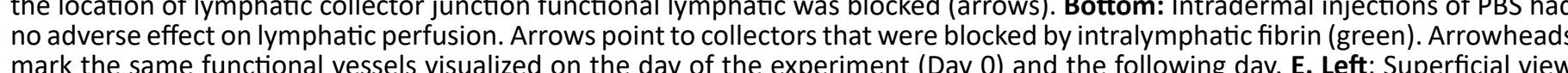
of the live dorsal skin of the ear after lymphangiography. Combination of all three pro-clotting factors in single treatment had no

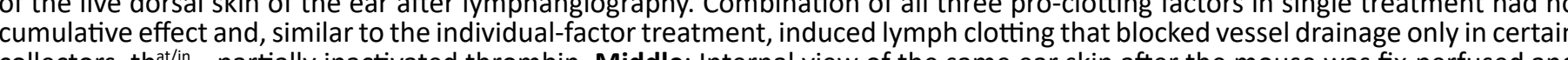

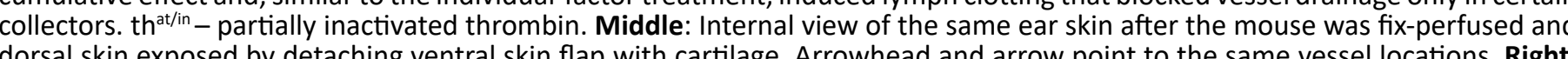

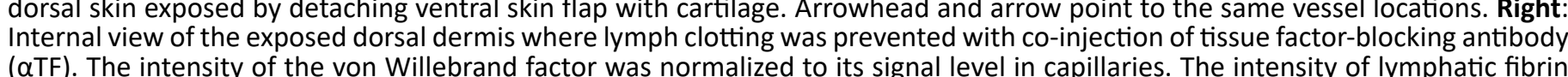
deposits was normalized to the signal level of clots at the sites of injection (*). Scale bar: A, C - 50um, D, E - 250um.collectors, and TF expression was reduced or absent on lymphatic endothelial cells. Arrows point to fibrin clots, arrowheads, MHCII-positive antigen presenting cells. L marks lymphatic collectors. TF intensity was normalized to the TF expression level of interstitial cells. IgGs, hence the TF signal can be appreciated only at the edges or outside the clot. Scale bar: $50 \mathrm{\mu m}$.

In contrast to blood circulation, where vessel occlusion of intratumoral lymphatics in most human cancers ${ }^{30}$. by thrombus leads to tissue ischemia and immediate However, fibrin as a provisional matrix undergoes rapid localized symptomatic pain, development of edema that digestion or fibrotic remodeling ${ }^{31}$ while pre-symptomatic follows lymphatic blockage can take a long time and tumor development is a chronic disease that can take till remann f pathe par lymph clots encapsulating the granuloma were found in by us lymphatic fibrosis in an experimental mouse model the chronic inflammation ${ }^{23}$, surrounding dead worms in of B16-F10 melanoma (Supplementary Fig. 1).

lymphatic filariasis ${ }^{24}, 25$ or occluding vessels in patients
lye transport of clottable lymph requires anchoring (he walls of lymphatic ymphedema ${ }^{27}$. These clots often undergo fibrosis, forming collectors but also creates an opportunity for lymphatic long-lasting intravascular intrusions in chronic diseases endothelium to change lymphatic patency depending on $23,25,27$. The tumor microenvironment is another typical tissue condition dynamically. Hence, we suspected that inflammatory pathology, where leaky blood vessels and lymphatic endothelium is critical to sustaining paten chronic inflammation should constitute ideal conditions lymphatic collectors but also that it could induce lymph for intravascular lymph clotting. Indeed, biomarkers of clotting by, e.g. TF expression alone or in collaboration tumor stroma, i. TF overexpression, interstitial fluid with leukocytes accumulating within lymphatics during hypercoagulability and stromal fibrin deposition ${ }^{28,29}$ inflammation. In that case, it should be possible to reverse correlate with dysfunctionality or complete occlusion potential anti-hemostatic properties of lymphatics with pharmacological inhibition of thrombomodulin, turning lymphatic endothelium into a pro-thrombotic surface with the expression of pro-thrombotic tissue factor (TF) or decellularization of lymphatic collectors, which not only removes the endothelial source of anti-thrombotic factors (thrombomodulin, plasminogen activators) but also expose negatively charged basement membranes, which laminin and collagen $I^{33}$ can activate intrinsic coagulation pathway ${ }^{34}$. We verified that challenge-induced formation of intravascular lymph clots primarily relied on the physiological state of the endothelium rather than the presence of infiltrating leukocytes. Finally, we showed that intact lymphatic endothelium is indispensable for the sustenance of lymphatic patency but not lymph and immune cell transport through collecting vessels. As a proof that anti-clotting function of lymphatic endothelium can be applied therapeutically, we showed that complete block of lymph transport to the draining lymph note block of lymph transport to the draining lymph node delayed immune response against subcutaneous allograft the lymph node to antigens lead to their ignorance by the immune system, a local treatment that can be used to prolong the survival of allografted cells or tissues without systemic and toxic immunosuppression.

\section{Results}

Induced TF expression on lymphatics leads to lymph clotting and obstruction of drainage

Here we analyzed the anti-clotting properties of lymphatics and the means to control them. In a normal physiological state the endothelium of lymphatic collector, similar to blood endothelial cells, express anti-thrombotic thrombomodulin receptor but do not express tissue factor (Fig. 1A). However, contrary to blood endothelium, lymphatic collector endothelium is not expected to encounter platelets, and consequently, they lack the intracellular stores of von Willebrand factor, the critical factor for platelet adhesion to the subendothelial collagen at the location of vascular wounds. Stimulation of de novo expression of tissue factor on lymphatic endothelial cells appeared as the most intuitive and feasible approach. We assayed the activation of intralymphatic coagulation in mouse skin by posttreatment intravenous injection of fluorescently labeled fibrinogen (Fig. 1B). If fibrinogen that leaked to the tissue was polymerized into insoluble fibrin, its fluorescent deposits could be detected with intravital microscopy of thin mouse ear or, after fixation, in the whole mount preparation with confocal optical sectioning.

TNF $\alpha$ and IL1 $\beta$ are cytokines known as potent inducers of tissue factor (TF) expression in vitro ${ }^{20,35}$ but only on subsets of blood endothelial cells in vivo ${ }^{36}$. Inflammatory stimulation of TF on the intima layer of blood vessels results in activation of the extrinsic pathway within vessel lumen and temporary, local vessel occlusion by thrombus. Similar in lymphatics, even in the absence of platelets, repeated intradermal injections of $\mathrm{TNFa}$ induced clot formation within draining collectors but only at discrete locations (Fig.1C). No cumulative effect observed when TNF $\alpha$ was co-injected with IL-1 $\beta$, and treatment produced a similar sparse distribution of clots within collectors. Activation of G-protein coupled receptor PAR-1 with thrombin acts synergistically with TNFa on TF expression on blood endothelium ${ }^{37}$ an e. Supprising, injection of $2.5 \mathrm{IU}$ throct to cyto sure from the site of inj oction but as expected led to away coagulation ca). A). However, we found that treatment of thrombin with (A) fuoride (PMSF) inactivated $80 \%$ of the enzyme (sixtimes times longer clotting time). Similar to cytokines reatment, recurrent intradermal injections of the mix of partially inactivated thrombin (thromb ${ }^{\text {in/ac }}$ ) induced the formation of intralymphatic clots within specific locations of lymphatic collectors without the bystander coagulation within blood vessels circulation (Fig $1 \mathrm{C}$ and Supplementary Fig 2B). Injections of thrombin diluted to $0.5 \mathrm{IU}$ concentration resulted again in coagulation within blood vessel over the lower surface of the skin but had no effect on intralymphatic clotting (not shown) suggesting that inactive thrombin had an effect on lymph clotting. Even though fibrin was deposited only in few collector branches (but always in at least one collector segment in

all 9 tested ears), their formation was sufficient to occlude lymphatic drainage from all lymphatics efferent to that collector (Fig. 1D). Against our expectations, there was no cumulative effect of combined treatment with cytokines and thrombin on lymph clotting and similar to injection of individual factors only discrete locations in collectors were functionally tors only discrete locations in collectors were pited with fibrin clots. Occlusion induce bartially inactivated thrombin (thromb 2A) or a mix of TNF $\alpha$ and Il-1 $\beta$ cytokines an

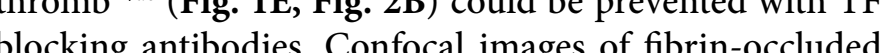
ymphatics stained for TF revealed that lym occluded collow cexpression of TF on lymphatic cose not induce lymph clotting directly but instead it activates certin cellusters in divergent endothelium of collectors. Heterogeneity of TF expression resembles the similar discontinuous pattern of CCL21 expression previously reported by our group ${ }^{38}$ but is also characteristic for blood endothelial cells where TF density on the cell surface varied strongly among TNF-alpha-stimulated endothelial cells in vitro ${ }^{20}$ and in vivo ${ }^{36,39}$. Combined treatment with cytokines and thrombin ${ }^{\text {in/ac }}$ had a cumulative effect on dendritic cells (DCs) immigration to lymphatics. In contrast, either due to faster transmigration, stimulated cell death or inhibition of migration of DCs to lymphatics, DCs were absent from intralymphatic cloths in triplefactor stimulated lymphatics but filled the lymphatic along the high TF expression zone in thrombin ${ }^{\text {in/ac }}$ treated skin (Fig. 2B). This lack of co-localization of intralymphatic DCs and lymph clots but also a weaker expression of TF (Fig. 2A inset) as compared to activated lymphatics indicated that lymphatic endothelium-derived TF was 


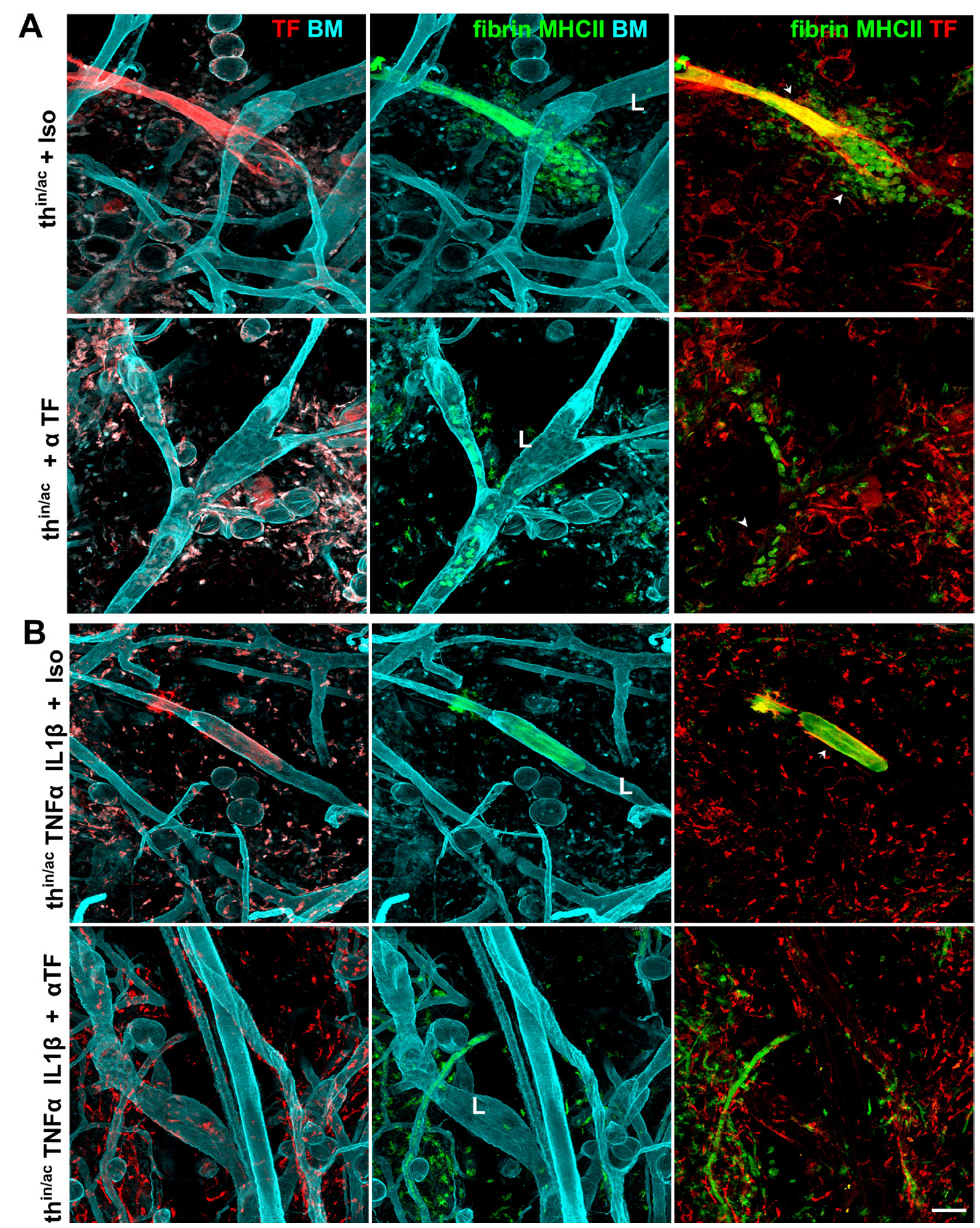

Figure 2. Intralymphatic coagulation induced by cytokine or partially-inactivated thrombin is dependent on tissue factor expression on lymphatic endothelium. Confocal whole-mount images of lymphatic collectors occluded by intralymphatic clots
ffter two consecutive intradermal injections with pro-clotting factors with isotype control or tissue factor blocking antibody (21E10) Basement membrane (BM) was stained with collagen IV, antigen presenting cells are stained with MHCII. A. Injection of partially nactivated (thrombinin/ac) together with isotype control antibody induced high expression of TF on discrete location of collectors vessels). Collectors that express a high level of TF were densely infiltrated with MHCII-positive antigen presenting cells. TF-blocking antibody ( ( TF) completely abolished clot formation but not clustering of antigen presenting cells within lymphatic collectors. B. Similar, in the presence of the isotype control antibody, mix of partially inactivated thrombin (thrombin in/c) and cytokines (TNFod
and II 1 3 ) induced fibrin clot formation in discrete locations within downstream collecting vasculature. In contrast to thrombin and IL1B) induced fibrin clot formation in discrete locations within downstream collecting vasculature. In contrast to thrombinit
ac alone, these clots were not infiltrated with MHCII-positive leukocytes. When $\alpha$ TF antibody was injected with the pro-clotting

mix, lymph clots did not form within lymphatic collectors, and TF expression was reduced or absent on lymphatic endothelial cells. Arrows point to fibrin clots, arrowheads, MHCII-positive antigen presenting cells. L marks lymphatic collectors. TF intensity was normalized to the TF expression level of interstitial cells. Note that lymph clots are autofluorescent in the red channel due to outside the clot Scale bar: $50 \mu \mathrm{m}$.

sufficient to induce clot formation. Inhibition of anti- endothelium ${ }^{39}$. PAR-1, however, has to be activated clotting pathway by thrombomodulin blocking leads to by thrombin proteolytic cleavage, hence inactivated instant dissipated intralymphatic lymph clotting. thrombin cannot initiate its signaling. On the contrary There is a low basal level of tissue factor-dependent thrombin binding by thrombomodulin is independent activation or 'idling' of the clotting system in normal on its enzymatic state when inhibited by small molecular individuals resulting in a constant, low level of formation inhibitors at thrombin active site ${ }^{42}$. Thrombomodulin of thrombin. In the absence of an anti-clotting mechanism, bound thrombin shifts its enzymatic specificity from the activation threshold of clotting cascade is crossed, fibrinogen to protein $\mathrm{C}$, which activation, in turn, leads and clotting cascade is initiated ${ }^{40,41}$. The necessity of co- to the destruction of co-factors V and VIII and inhibition injection of mixed active and inactive thrombin to initiate of clotting process ${ }^{18}$. Hence, blocking thrombin binding lymph clotting suggested that inactivated thrombin served sites on thrombomodulin can inhibit the whole antia role in activation of lymph cloting. Thrombin bas two clotting pathay. Treatment of thromin with the potent, types of receptors on

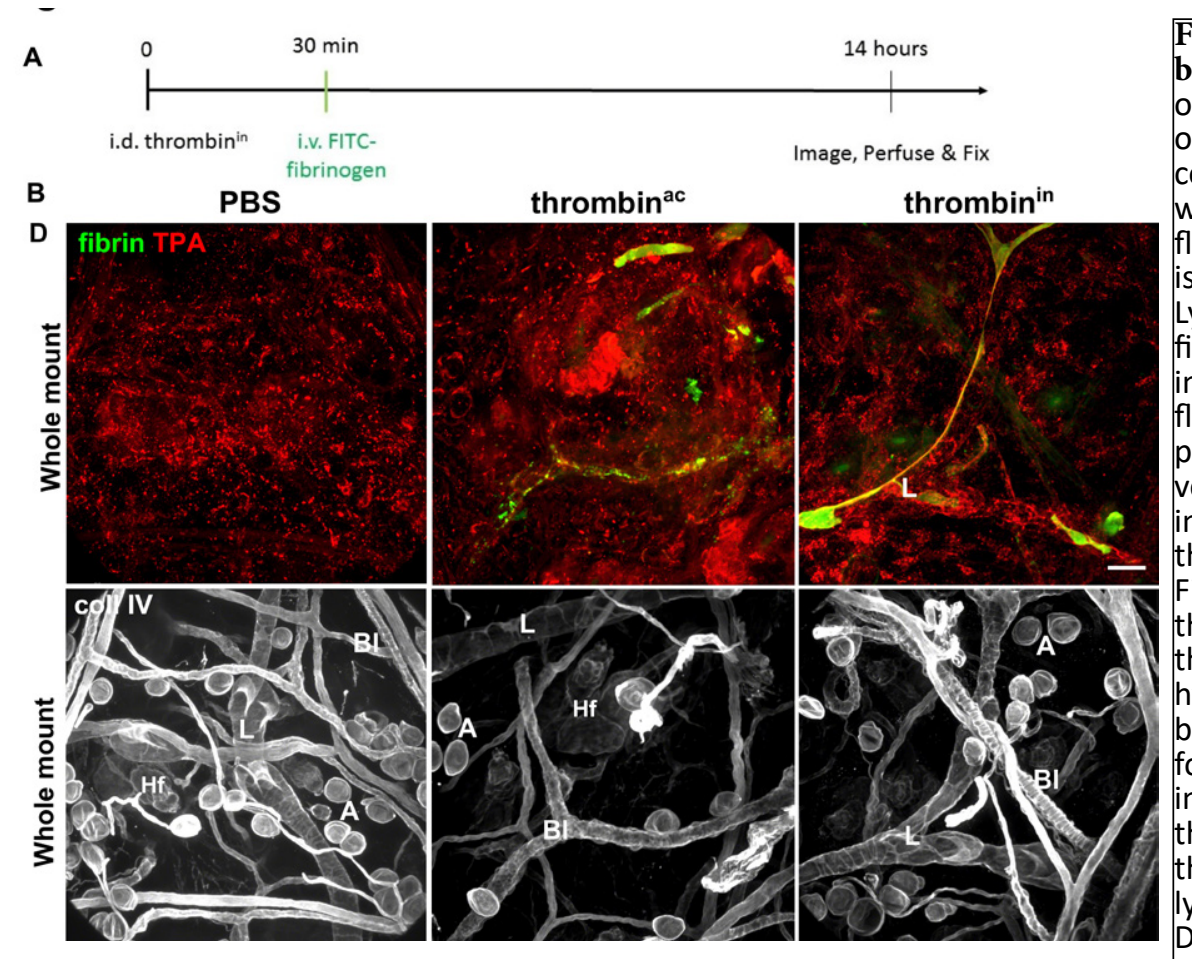
lymphangiography are bridged by green fibrin deposits, indicating an incomplete occlusion. In contrast to continuous fibrin clots lymphatic collectors but had no effect on plasma coagulation in blood vessels (Bottom thrombinin). C. Higher magnification confocal images 5 hours after the second injection of compounds indicated in B. Collagen IV stained lymphatic collectors (L) and mice, fibrin slots were not formed away from the injection site. CD 31 showed normal cell-cell junctions between lymphatics (PBS). Active thrombin blocked blood circulation, trapping autofluorescent erythrocytes (yellow) within blood vessels (BI) around the injection site. Rare intralymphatic clots (L) could be found only around the thrombin ${ }^{\text {ac }}$ injection site (Thrombinac). In the Thrombin injected skin, discontinued fibrin clots in remote lymphatic collectors were observed. D. Top. 16 hours after injection of PBS, tissue plasm Thrombinac injection. In the skin injected with Thrombinin ${ }^{\text {n }}$. TPA strongly labeled collecting lymphatic vessels filled with residual,

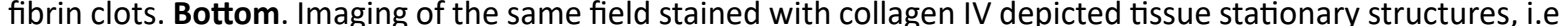
blood vessels, lymphatics, and adipocytes. A-adipocytes, L-lymphatic collectors, Bl-blood vessels, M-macrophages, Hf-hair follicle The intensity of PAA was normalized to its signal level in interstial cells. The intensity of lymphatic fibrin deposits was normalized

of the enzyme ${ }^{43}$ and in our hands, it prolonged 400-times inactivated thrombin-induced lymph clotting in most times the clotting time. A single injection of saline had segments of draining lymphatic collectors. However, due no effect on lymph clotting or dextran drainage (Fig 3A- to incomplete lumen occlusion and rapid dissolution of B) and draining lymphatics preserved normal cell-cell these clots, intralymphatic clots only partially inhibited the junctions. Fully active thrombin-induced coagulation lymphatic drainage, the blockage could be overcome with within blood vessels at the site of injection but had no pressurized syringe injection. Inactive thrombin injected effect on lymph clotting in remote lymphatics. APMSF- skin, intralymphatic fibrin clots could be found only 


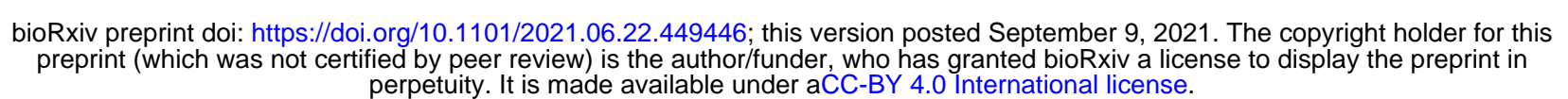

around the site of the injection together with blood vessel expression. A similar mechanism might be responsible clots entrapping autofluorescent erythrocytes within blood for fast clearance of lymph clots in inflammatory factorvessels (Fig. 3C). Fast dissolution of intralymphatic clots treated lymphatics where we also observed a high level of (after injection of inactive thrombin, thrombin ${ }^{\text {in }}$ ) could be TPA expression within stimulated lymphatics (not shown) explained by increased expression of tissue plasminogen Intradermal clot deposited in the skin outside blood activator by lymphatic endothelial cells. Indeed, 16 hours vasculature remains within the skin in roughly unchanged after injection of thrombin in , lymphatic collecting vessels shape for at least 24 hours (Fig. 4A). In contrast, emerged from other tissue structures by an elevated level thrombinac-induced blood vessel clots were almost entirely of TPA expression (Fig. 3D). Active thrombin (thrombin ${ }^{\mathrm{c}}$ ) cleared from clot formation by thrombomodulin blocking at the site of injection-induced larger tissue damage lost its dependency on TF after tissue pre-injection with and robust activation of TPA in various tissue cells, i.e., TF-blocking antibodies. This possibility was excluded as adipocytes, macrophages and also blood vessels, making we showed that mock pre-injection or injection with TFthese structures to stand out from surrounding tissue. In blocking antibodies and subsequent injection of skin with regular non stimulated tissue TPA was mostly expressed TF-blocking antibodies and thrombin ${ }^{\text {in }}$ did not inhibit by macrophages, but with the exception of hair follicles, no the circulation. Re-injection of saline and thrombin ${ }^{\text {ac }}$ restationary structures could be identified by distinctive TPA created clots in dermis and blood vasculature, respectively.

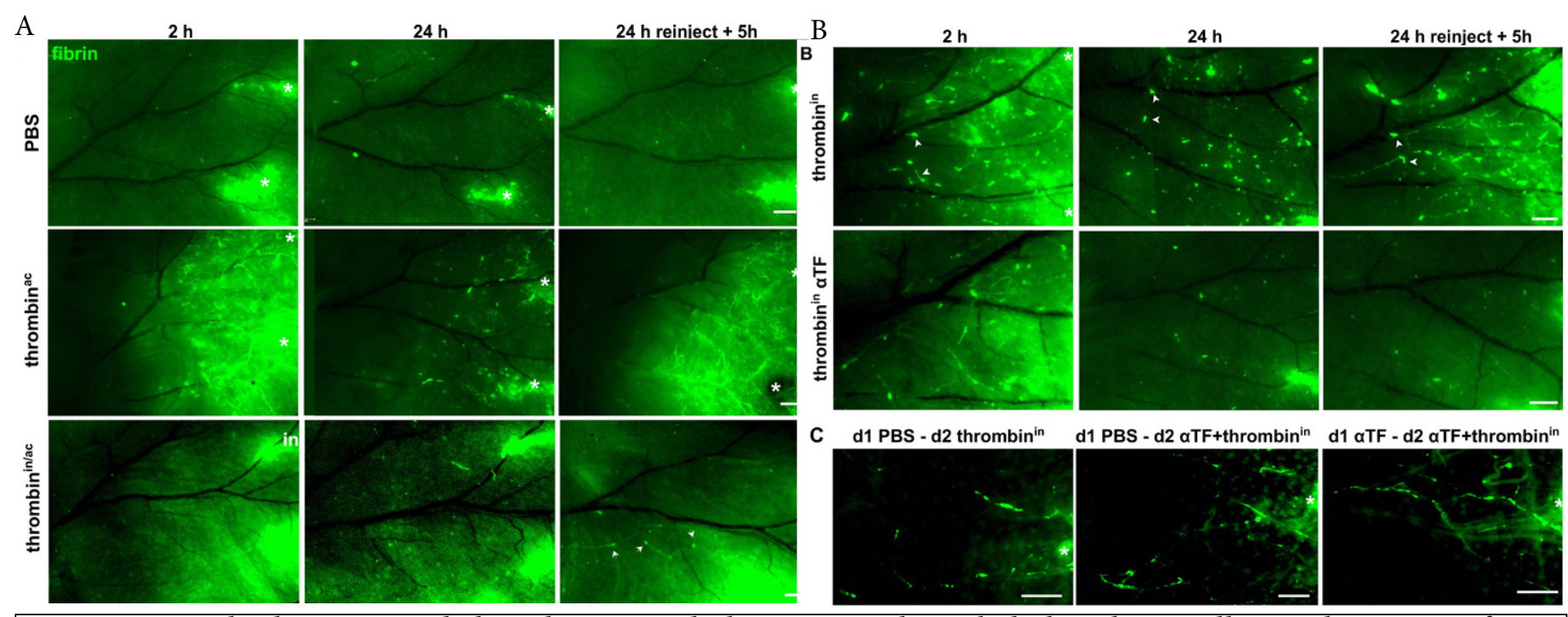

Figure 4. Completely inactivated thrombin instantly but transiently occlude lymphatic collectors by a tissue factor ndependent mechanism. Intravital imaging of fluorescent fibrin clot formation within the ear skin vasculature. Fluorescen
fibrinogen was administered i.v. immediately after $2.5 \mu \mathrm{\mu l}$ intradermal injection of indicated compounds in two adjacent injection sites on a single ear dorsal dermis. In all images, lymphatics drain from the right (distal) to the left (proximal). The functional blood vessels appear dark in all images as tissue was fixed by perfusion to clear the vessels from non-coagulated fibrinogen. A. Top

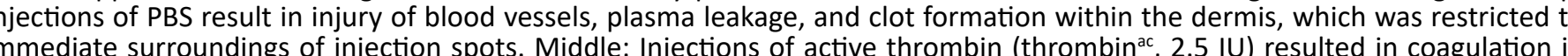
mmediate surroundings of injection spots. Middle: Injections of active thrombin (thrombinac, $2.5 \mathrm{IU})$ resulted in coagulation in
blood vessels, blood vessel leakiness, and fibrin clotting within the dermis in the area of the ear where injection edema formed. Itravascular and interstitial clots were largely cleared within 24 hours after the injection, however, they could be re-created
with another intradermal injection of active thrombinac. Bottom: Similar to PBS injections, a single dose of partially inactivated with another intradermal injection of active thrombinac. Bottom: Similar to PBS injections, a single dose of partially inactivated
thrombin (thrombin in/ac) caused leakage of plasma and intradermal fibrin clotting around the site of injection but had no effect thrombin (thrombinin/ac) caused leakage of plasma and in of thrombinin/ac 24 hours later induced the formation of intralymphatic clots at discrete lymphatic locations, downstream to the sites of injection. Arrows point to single intralymphatic clots defined by their sharp edges, in contrast to the whole lymphatic that is visible due to non-coagulated fibrinogen labeled lymph. B. Ears after
njection with thrombinin inactivated with APMSF (400-fold decrease in activity, thrombinin). Top: Single dose of thrombinin (conjected with irrelevant rat antibodies) resulted in rapid formation of fibrin clots within most draining lymphatic collectors, which were mostly cleared 22 hours later. Reinjection of thrombinin re-created clotting pattern within lymphatics. Arrowheads point th of tissue factor-blocking ( $\alpha$ TF) antibodies and thrombinin did not inhibit instant intralymphatic clot formation, however reinjection of tissue factor-blocking ( $\alpha$ TF) antibodies and thrombin in did not inhibit instant intralymphatic clot formation, however reinjection
of $\alpha \mathrm{TF}$ and thrombinin 24 hours later did not induce the formation of intralymphatic clots. C. Neither pre-injections with PBS injection injury) nor $\alpha$ TF antibody (day 1 ) inhibited intralymphatic clot formation induced with thrombinin or with thrombinin and aTF co-injection on day 2 at 24 hours after the first injection. Images were taken from ears fixed 5 hours after the second injection he intensity of CD31 was normalized to its signal level in the lymphatics draining control region. The intensity of lymphatic fibri

In contrast, the effect after the first injection of thrombin ${ }^{\text {in/ } /}$ endothelial cells upregulate production of TPA (Fig. 3D). ac, partially inactivated with PMSF resembled injection of In that scenario, idle extravascular TF activity or contact saline. Only the second injection Also, a large number of activation pathway initially suffice for the accumulative rounded inflammatory cells infiltrated $t$ of thrombin ${ }^{\text {in/ac }}$ lymph clotting and become insufficient in the presence of 24 lymph clotting (Fig. 4C). Instead, it seems likely that high levels of plasmin.

initial activation of clotting pathway leads to sensitization Noxious agents kill endothelium that leads to lymph to the extravascular TF by shifting the intralymphatic

hemostatic balance to fibrinolytic, where activated leads to localized sterile inflammation that should have hours after the first injection induced lymph clotting in multifactorial pro-hemostatic effects on the transported discrete and remote to injection sites lymphatic segments. lymph $^{44}$. First, the elimination of thrombomodulin anti- Thrombin $^{\text {in }}$ completely inactivated with APMSF induced clotting mechanism inherently linked to lymphatic dissipated fibrin clotting in most collectors already 2 hours endothelial cells promotes amplification of contact- after the injection. Similar to in the case of blood vessels, dependent pathway on exposed RNA and polyphosphates most intralymphatic clots were cleared 22 hours later (Fig. from dying cells $s^{45}$. Also, decellularization of the lymphatic 4B). First co-injection of thrombin ${ }^{\text {in }}$ with TF-blocking surface should prolong the occlusion of lymphatics antibody did not inhibit intralymphatic clot formation. surface should prolong the occlusion of lymphatics antibody did not inhibit intralymphatic clot formation.
as it eliminates the source of endothelium-derived Unexpectedly, re-injection of TF-blocking antibodies 22 as it eliminates the source of endothelium-derived Unexpectedly, re-injection of TF-blocking antibodies 22
tissue plasminogen activator ${ }^{46}$ that is a cause of the fast hours later completely blocked thrombinin-dependent clearance of intralymphatic clots from TF-expressing or intralymphatic clot formation, suggesting that activated thrombomodulin blocked lymphatics (Fig. 3E). Finally, monocytes that could directly induce lymph clotting by sterile inflammation leads to infiltration of TF-expressing extrinsic pathway ${ }^{47}$. To induce sterile inflammation of hydroxyl and hydroperoxyl radicals from mitochondria- collecting vessel endothelium we choose agents that have derived hydrogen peroxide and it is used in TF-independent a direct toxic effect on living cells, a cell lysing detergent blood vessel thrombus formation models ${ }^{48}$. Intradermally Triton-X-100 (Triton) and $\mathrm{FeCl}_{3}$, a catalyst generating

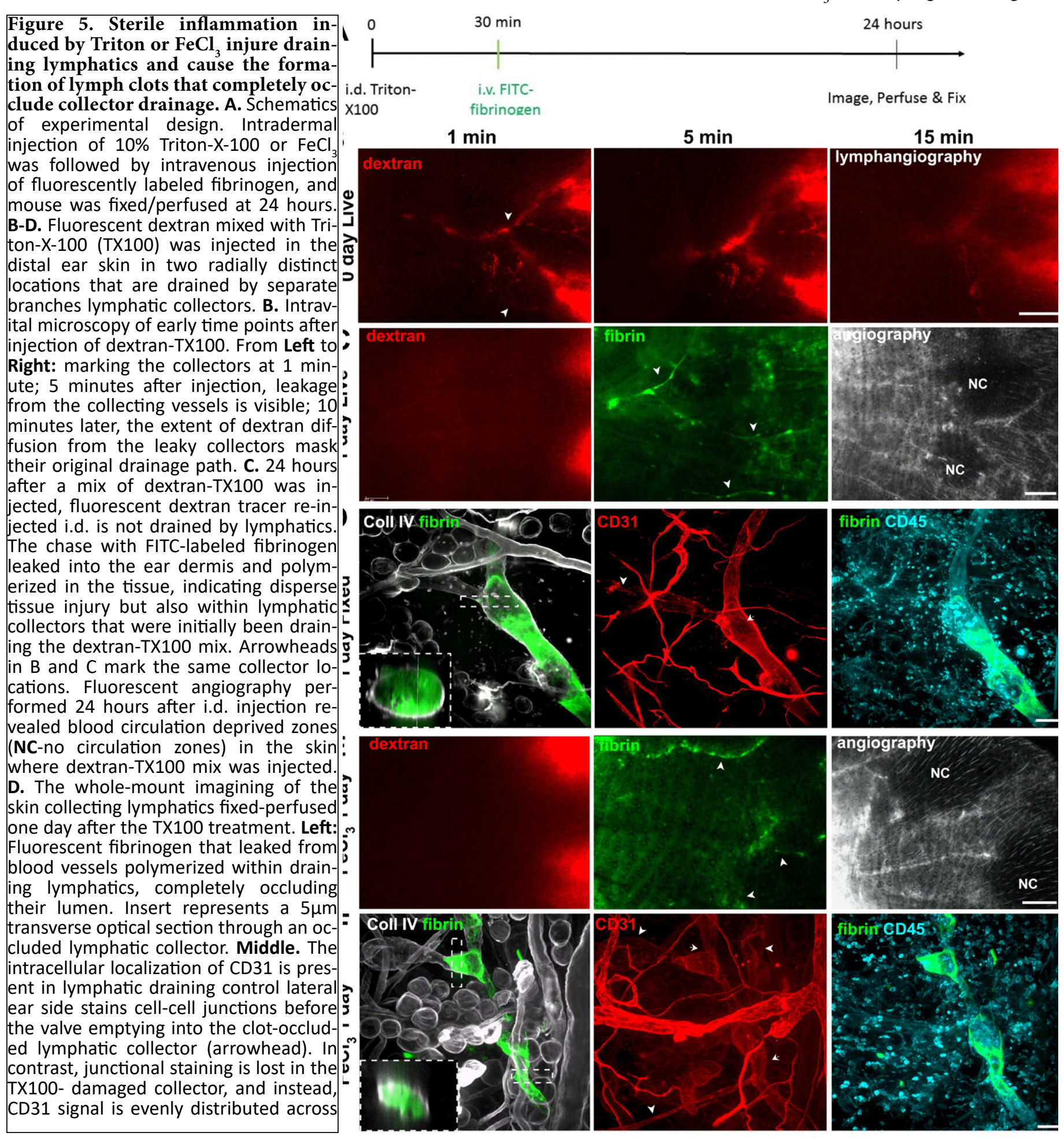


injected dextran mixed with $1 \%$ detergent Triton-X-100 drainage from the whole lymphatic tree. Including intact was drained initially by collecting lymphatics, to be leaked vessels draining the lateral side of the ear skin which are out of the lymphatic collector into the remote tissue only connected to the occluded lymphatic (Fig. 5D and F). Thi minutes later (Fig. 5B). Lymphangiography repeated approach unspecifically exerts bystander toxicity to any on the next day showed that draining of cell lysis agent cell type present at the site of injection. Also, toxins canno blocked the tissue drainage completely and instead be inactivated and instead they can freely diffuse outside lymphatic collectors were filled with continues lymph the collectors potentially injuring cells in remote locations. clotting (Fig. 5C). As expected, angiography performed Instead, photodynamic therapy (PDT) offers a solution to day after intradermal injection of Triton showed that all these limitations. PDT is a combined physicochemical blood circulation was lost around the injection spots treatment confirming Triton in vivo universal toxicity and capability acts as proto-toxin, which is activated and destroyed chent assures that the toxicity of free radicals difusing on short filled conpletely lumen of the distances (up to $7 \mu \mathrm{m}$ ) will be strictly limited to collectors lost the fine organization of cell-cell junctions (Fig. 5D). associated cells ${ }^{49}$. Large, $100 \mathrm{~nm}$ in diameter Visudyne However, the Triton toxicity was limited to the collectors liposome enclose an active compound, a small molecula specifically draining detergent as afferent lymphatic verteporfin, therefore their diffusion potential in the tissue draining control region but joined to the fibrin occluded was minimal. Practically, Visudyne ${ }^{\circ}$ liposomes were eithe collector vessels, but also blood capillaries surrounding directly injected to the lymphatic collectors or retain in occluded lymphatics, preserved their cellular organization. the skin interstitium. To avoid direct toxicity to the dermis Already one day after the treatment surrounding tissue that could result in enhanced trafficking of immune cells and fibrin-occluded lymphatics were populated by from the afferent injury site, the injection spots were rounded inflammatory cells. Different mechanism of painted black and covered with foil immediately after the $\mathrm{FeCl}$ toxicity that is dependent on free radical generation injection only leaving the exposed collectors. We reached 98\% of the reproducibility of the anti-lymphatic treatment roment ( and deposition of a feric chich of ferric chloride resulted in complete cessation of blood allowed us to eliminate mice with incompletely or poorly circulation around dermis injection sites. Fibrin clots filled injected collectors. The routine anti-lymphatic treatmen completely lumen of lymphatics draining ferric chloride, occluded all collectors draining peripheral dorsal ski which also lost its fine CD31-delinigthed junctional within 24 hours after PDT with continues lymph clots organization (Fig. 5F). Likewise Triton, ferric chloride (Fig. 6B). Likewise injection of directly-acting toxins, toxicity was limited to draining lymphatic as lymphatics Triton or ferric chloride (Fig. 5), and in contrast to TFdirectly connected to a fibrin-occluded collector but stimulation (Fig. 1-2) or thrombomodulin blocking (Fig. draining lateral (control) skin and surrounding blood 3-4), PDT always resulted in the formation of continuous capillaries preserved their cellular arrangement. Also, a intralymphatic fibrin clots in all vessels that drained the dermis in the draining region and crowded within the toxins. PDT resulted in much lower collateral damage to the tissue at the injection site that was manifested Specific decellularization of remote collectors leads to in minimal leakage of fluorescent plasma into a tissue complete and long lasting clot formation and lumen (cos occlusion

Injection of general toxins envisaged the importance of anti-clotting properties of the endothelium from lymphatic collectors. It also pictures ease and specificity whe can beted, additionally proving death deduced from degeneration of cellular junction was specific to endothelial lining coming in direct contact with a photosensitizer, leaving afferent vessels draining lateral side of ear dermis intact (Fig. 6D). Basement membrane that supports endothelium and muscle cells that blocking of only a few lymphac collectors ceases the of lymphatic collectors (Fig. 6D), remained as a scaffold

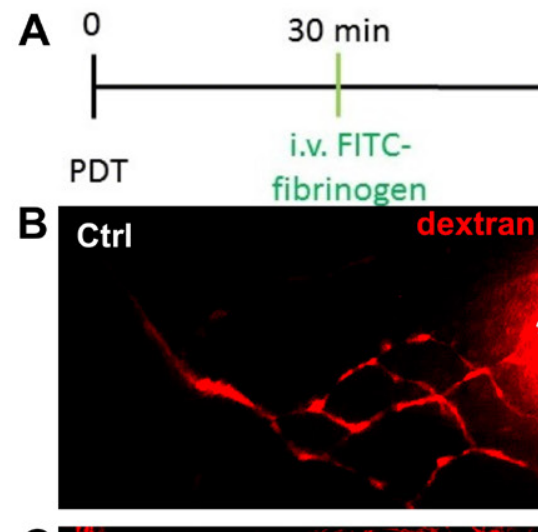

$\geq 24$ hours

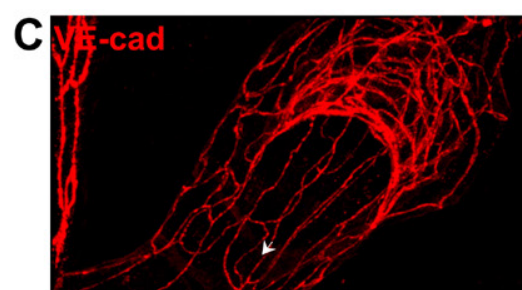

Crrl



Image, Perfuse \& Fix
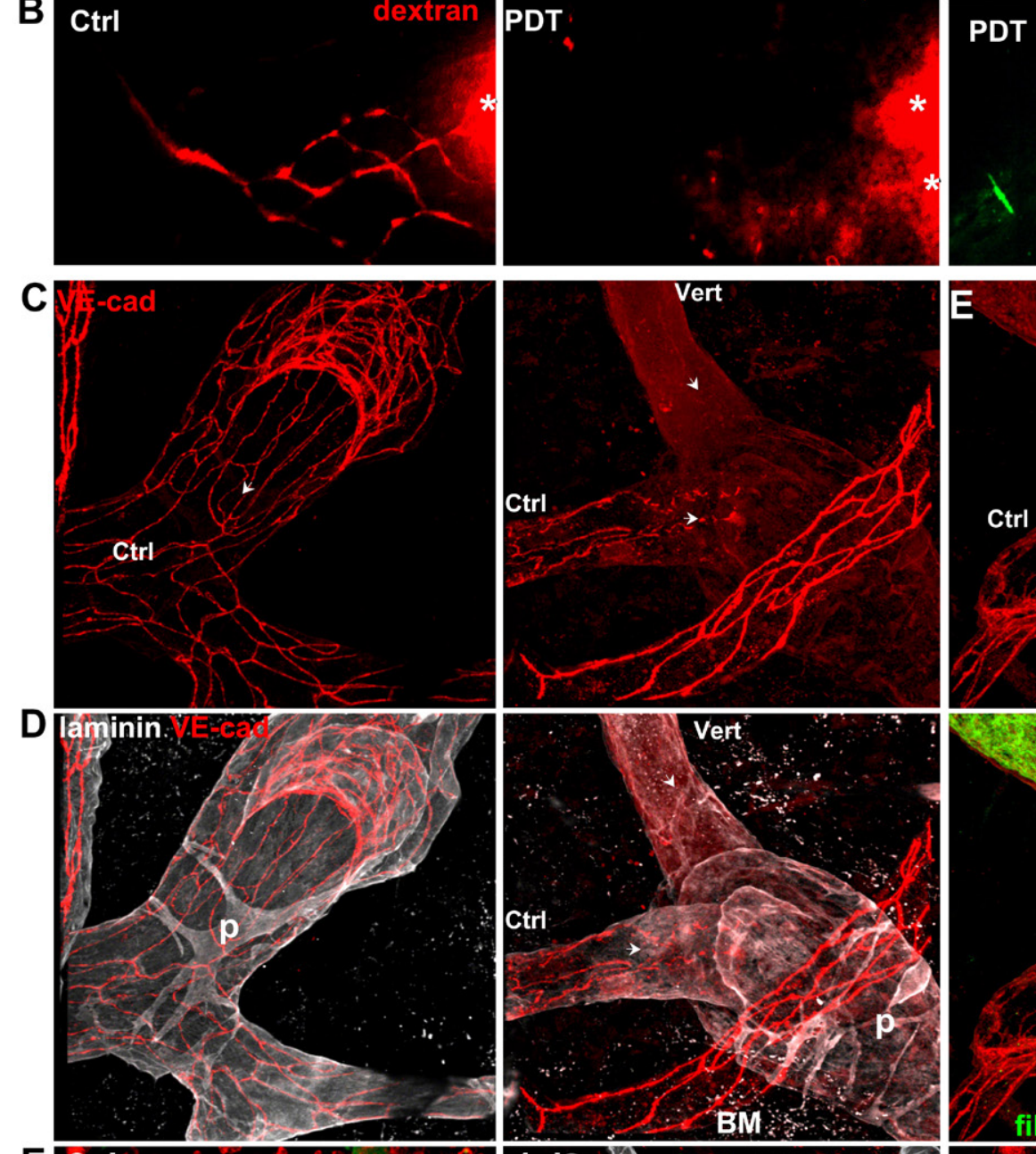

E day

fibrin $\mathrm{CD}$ Figure 6. Specific decellularization of lymphatic collectors leads to complete and long-lasting lumen occlusion. A.
Schematics of experimental design. Endothgelium was stripped from lymphatic collectors with a photodynamic therapy (PDT)
Specifically, intradermal (i.d.) injection of photosensitizer verteporfin Visudyne ${ }^{\oplus}$ was followed by collector imaging (verteporfin ymphangiography) and subsequent irradiation of dorsal ear skin. 20-30 min after PDT, fluorescently labeled fibrinogen was injected ntravenously, and ear lymphatics were imaged the following day. The mouse was fixed/perfused on the indicated day for whole mount imaging. B. Intravital microscopy, C-F-confocal imaging of whole-mount skin preparations. B. Left: Saline injection and epeated lymphangiography had no effect on collector perfusion in the control ear. Middle: PDT leads to occlusion of lymphatic
ollectors, which stop draining i.d. injected fluorescent dextran. Right: The same collector networks become occluded with 


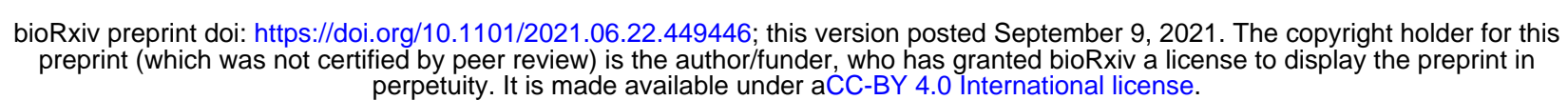

insoluble fibrin that leaked from blood vessels. C-E. Whole-mount imaging. Left: VE-cadherin is localized at cell-cell-junctions in

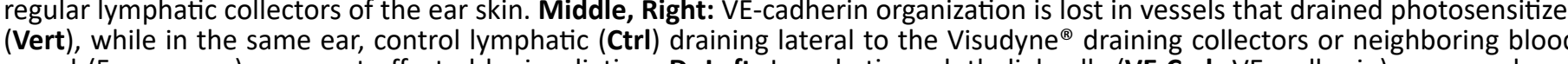
vessel ( $5 \mu \mathrm{m}$ away) were not affected by irradiation. D. Left: Lymphatic endothelial cells (VE-Cad-VE-cadherin) were enclosed Morphologically unaffected cell-deprived basement membrane tubes- 'ghost vessels'- one day after PDT. BM scaffold was preserved in PDT-decellularized collectors. Right: Ghost vessels were filled with fibrin clots that blocked the whole lymphatic umen. Insert shows $5 \mu \mathrm{m}$ cross-section of the fibrin-occluded collector. Frequently, basement membrane particles could be foun trapped within a clot (arrowhead). E. In addition to intralymphatic lymph clotting, ghost vessels become infiltrated with TGF 政 The intensity of CD 31 was normalized to its signal level in the lymphatics draining control region. The intensity of lymphatic fibrin

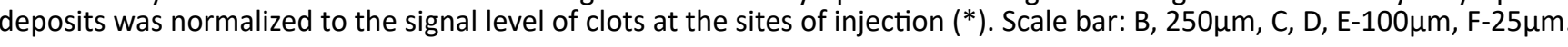

where lymphatic clots formed occluding the lumen of "ghost vessels" (Fig. 6D), named after similar blood vessel-derived structures that were described earlier by Inai ${ }^{50}$. Even though the basement membrane of collecting lymphatics remained intact, some degeneration occurred as BM particles could be found trapped within the fibrin clots (Fig. 6D, insert). Fibrin-occluded lymphatics wer densely infiltrated by rounded neutrophil-like immune cells from day two after PDT. Leukocyte clusters persisted at the cone of advancing endothelial cells that regenerated ghost vessel (Supplementary Fig. 3), possibly providin directional and stimulating cues by the release of, e.g. TGF (Fig. 6E) to re-growing endothelium ${ }^{51}$. In contrast to fibrin deposits within ghost vessels, which began to clear from day 4 onward, massive leukocyte infiltration persisted throughout the regeneration period of lymphatics even though (Fig. 6E). Fibrin deposits were eventually either cleared or replaced with abnormal non-sheet-like masses of basement membrane matrix, containing laminin,(Fig. 6F), collagen IV, collagen III and tenascin C (not shown). Basement membrane deposits eventually evanesced from vessels approximately by day 9 , which coincides with lymphatics re-gaining their drainage functionality $\left({ }^{49}\right.$ and Fig. 10b). Interestingly, similar intralymphatic matrix deposits, composed of collagen type I and IV we found within peritumoral collecting lymphatics in implanted dermal B16-F10 melanoma, which was paralleled by the abnormal, discontinued organization of lymphatic endothelial cells. (Supplementary Fig. 1).

Endothelial cell death precede lymph clotting and is independent on tissue factor

We could not find even a theoretical mechanism explaining why killing of lymphatic endothelial cells but not its basement membrane scaffold should lead to occlusion of collectors without implication of secondary mechanism, namely lymph clotting. This also suggests that the primary function of lymphatic collectors could be the control of lymph fluid state.

Live imaging of skin collector vessel endothelium expressing Tomato-fluorescent protein under Proxl promotor, with basement membrane stained for collagen IV showed that irradiated cells draining prototoxin Visudyne ${ }^{\circ}$ died between 1 and 3 hours after PDT (Supplementary video 1, Fig. 7A). Vertical afferent (Sumphatic collector served as internal control for afferent dependent bleching and photoxicity and showed dependent bleaching and phototoxicity and showed that only prior laser irradiation of drained photosensitizer
but not subsequent fluorescence imaging evoke death of lymphatic endothelium. As expected, there was no

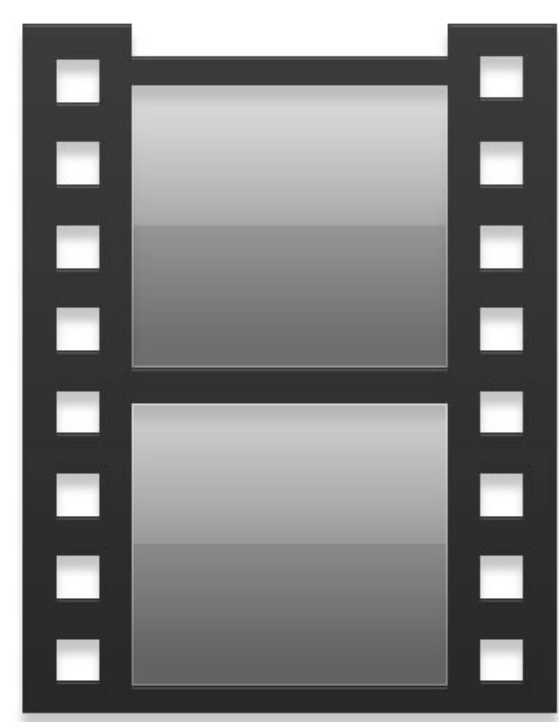

Video 2. Concurrent necrosis and apoptosis of B16-F10 cells grown for one week in the mouse ear. Single cell divided but the two daughter cells die by apoptosis (top cell breaks into
small apoptotic bodies which intensity is not different to the mother cell) and necrosis (bottom cell "explode," the GF
fuorescence diffuse out in $1-2$ frames of the 15 fps movie). The color gradient, from high-contrast white to green representing the weakest signal was chosen for the clarity of
the visualization. The real-life duration of the video is 2 hours.
The video frame rate is $15 \mathrm{fps}$.

the majority of endothelial cells treated with PDT diffuse their cytoplasmic proteins, the more informative indicative of the final stage of necrotic cell death (Suppl video 3). Despite that collector's endothelium dies off within 2.5 hours after PDT vessel occlusion started not earlier than 3 hours after PDT (Fig. 7B), which is in agreement with

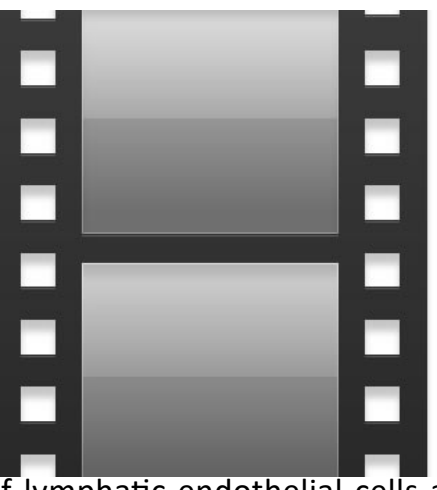

Necrotic death of lymphatic endothelial cells at the level of the valve 2 hours after photodynamic therapy. Propidium iodide, a membrane impermeable DNA-binding agent labels nuclei of necrotic cells. The real-life duration of the video is 8 minutes. The video frame rate is $4 \mathrm{fps}$.

our previous results ${ }^{49}$. The cessation of dextran drainage between 3 and 5 hours after PDT correlated with increased deposition of fibrin within collectors that reached its maximum 7 hours after PDT. As compared to blood vessel occlusion, the process that is completed within minutes after the injury, occlusion of lymphatic collector after PDTinduced decellularization was a slow process that took at least an hour and began with fibrin deposition on the wall of decellularized lymphatic and continued toward the center of the vessels (Suppl video 4 ). contrast to lymph clotting provoked by cytokinesJllularization cou

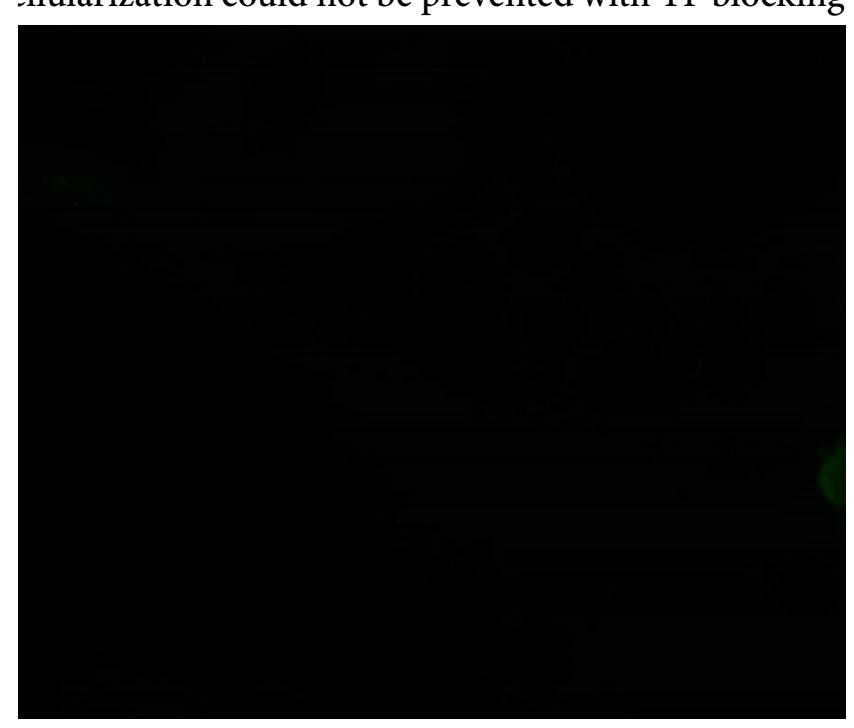

Video 4. Fibrin deposition on the wall of collecting lymphatic 作

intravenous injection of FITC-fibrin PDT and 1.5 hours after 列 are the effect of surgical exposition of dorsal dermis lead cased generalized leakage of plasma from vessels leading to
an increase in fibrinogen fluorescence outside blood vessels. Immediately after PDT mouse ear dorsal skin was exposed, and dermis was stained with collagen IV (not shown) to identify draining lymphatic vessels based on their characteristic morphology. The real-life duration of the video is 2 hours. The nfiltration of leukocytes into decellularized lymphatics despite the fact that antibody were present in the tissue two days after the treatment. Most importantly, we confirmed our previous observation that there is a temporal gap between endothelial cell death and lymphatic occlusion which indicated that at least during this lag perioc decellularized lymphatics were able to drain fluid withou a presence of endothelial cells.

Massive infiltration of antigen presenting cells and T-cells begin after lymphatic occlusion and persis until their regeneration

Cessation of drainage after lymphatic decellularization i directly associated with the formation of intralymphatic lots of fibrin inside collectors but also massive nfiltration of leukocytes (Fig. 5E). Here we asked if th atter phenomena could participate in the occlusion of ymphatics. In addition, we wanted to know what cell ypes are represented in the immunological clusters that form within lymphatics.

In contrast to fast infiltration of leukocytes into ghos lymphatics after intradermal injection of directly toxic compounds, Triton or ferric chloride, milder hour lag phase and during that time leucocyte presence 6A). Surprisingly, labeling of muscle cells smooth muscle actin with mouse monoclonal antibody revealed hat intralymphatic clots could be directly stained with detection anti-mouse IgG antibody indicating that mouse 


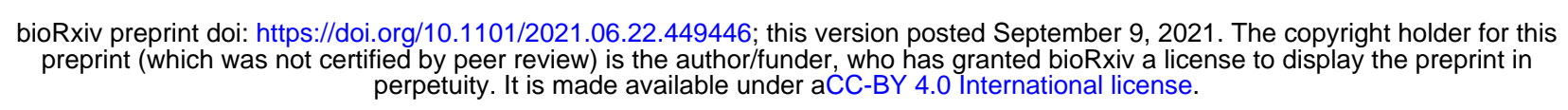

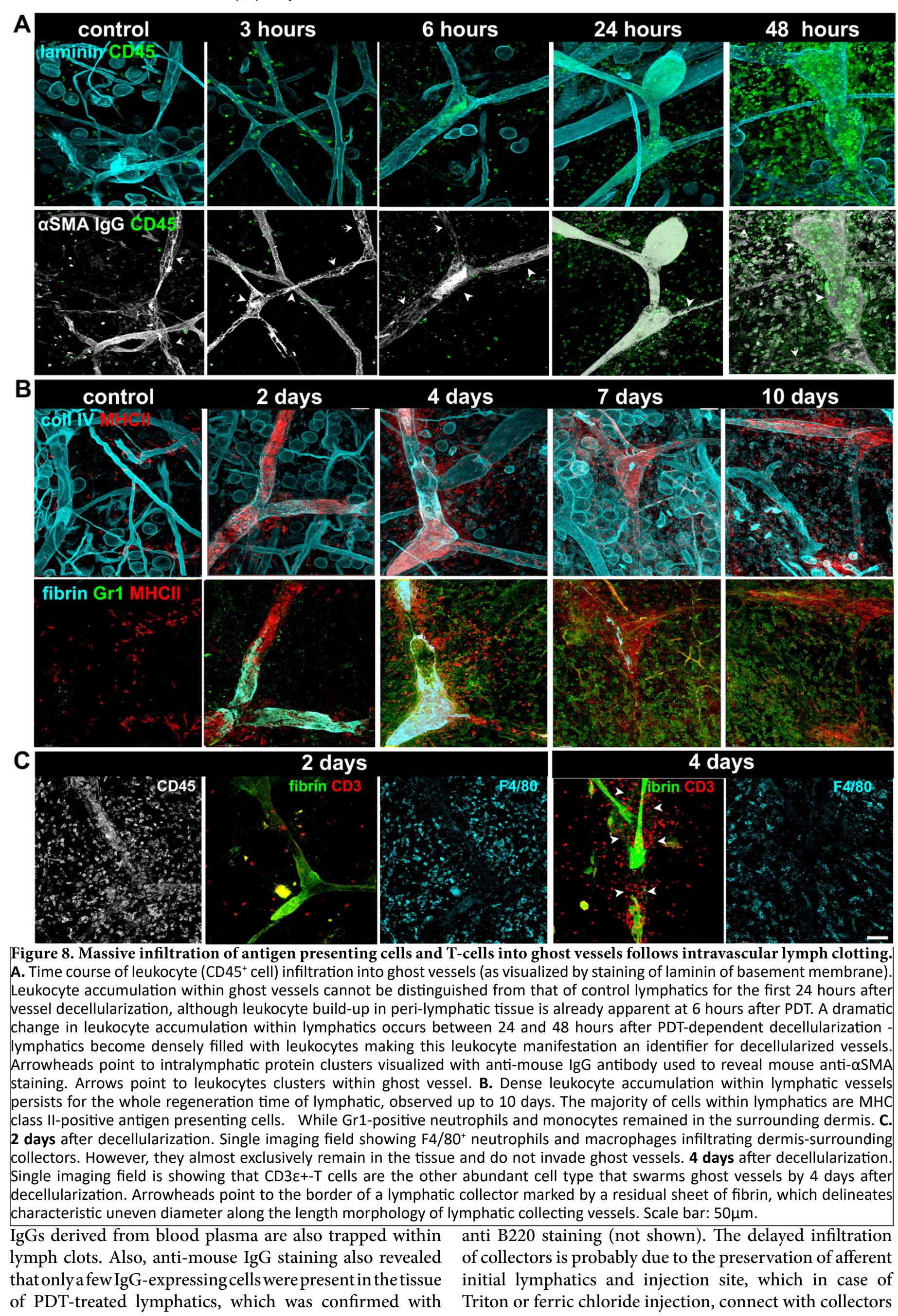



B PD
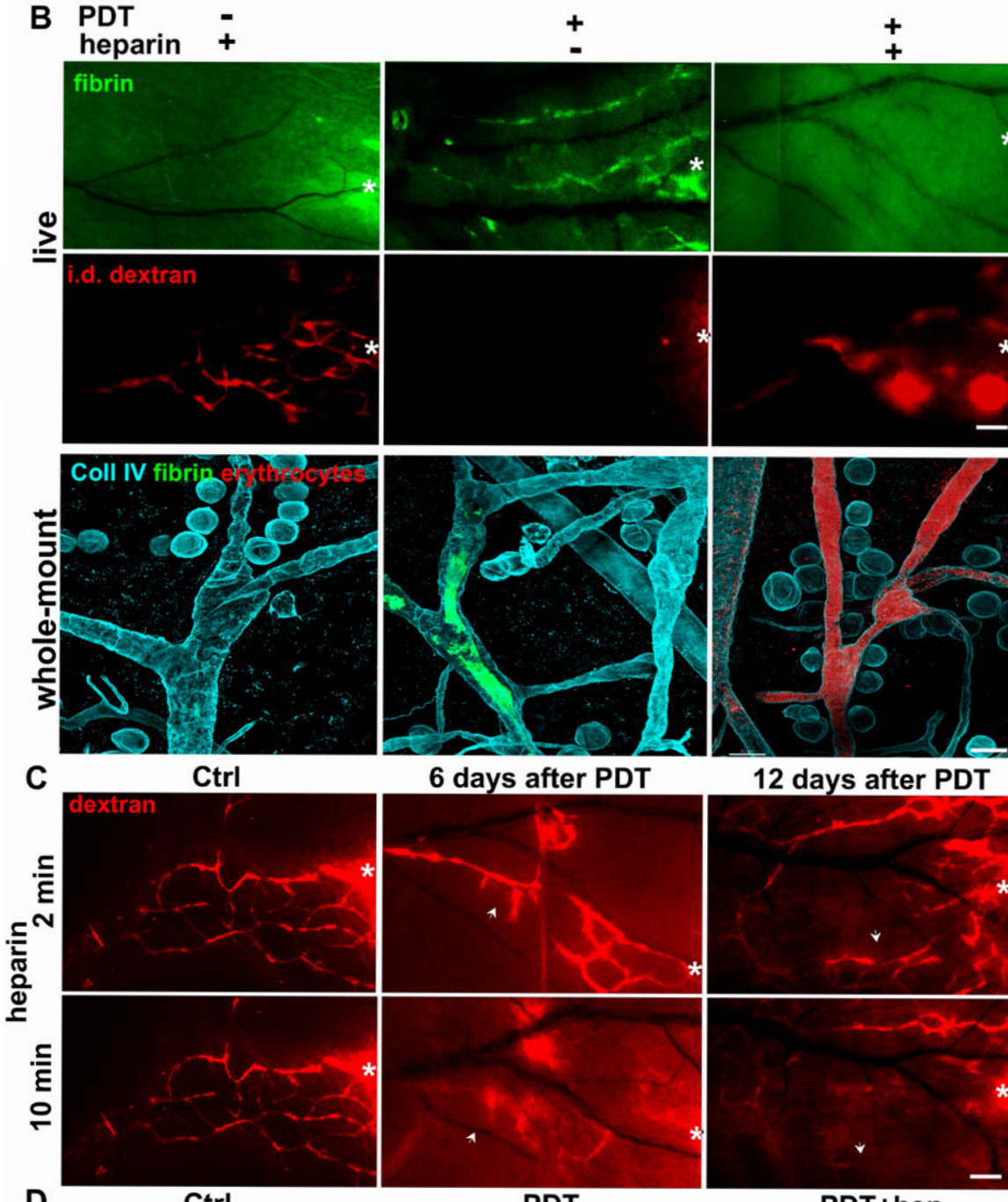

6 days after PDT
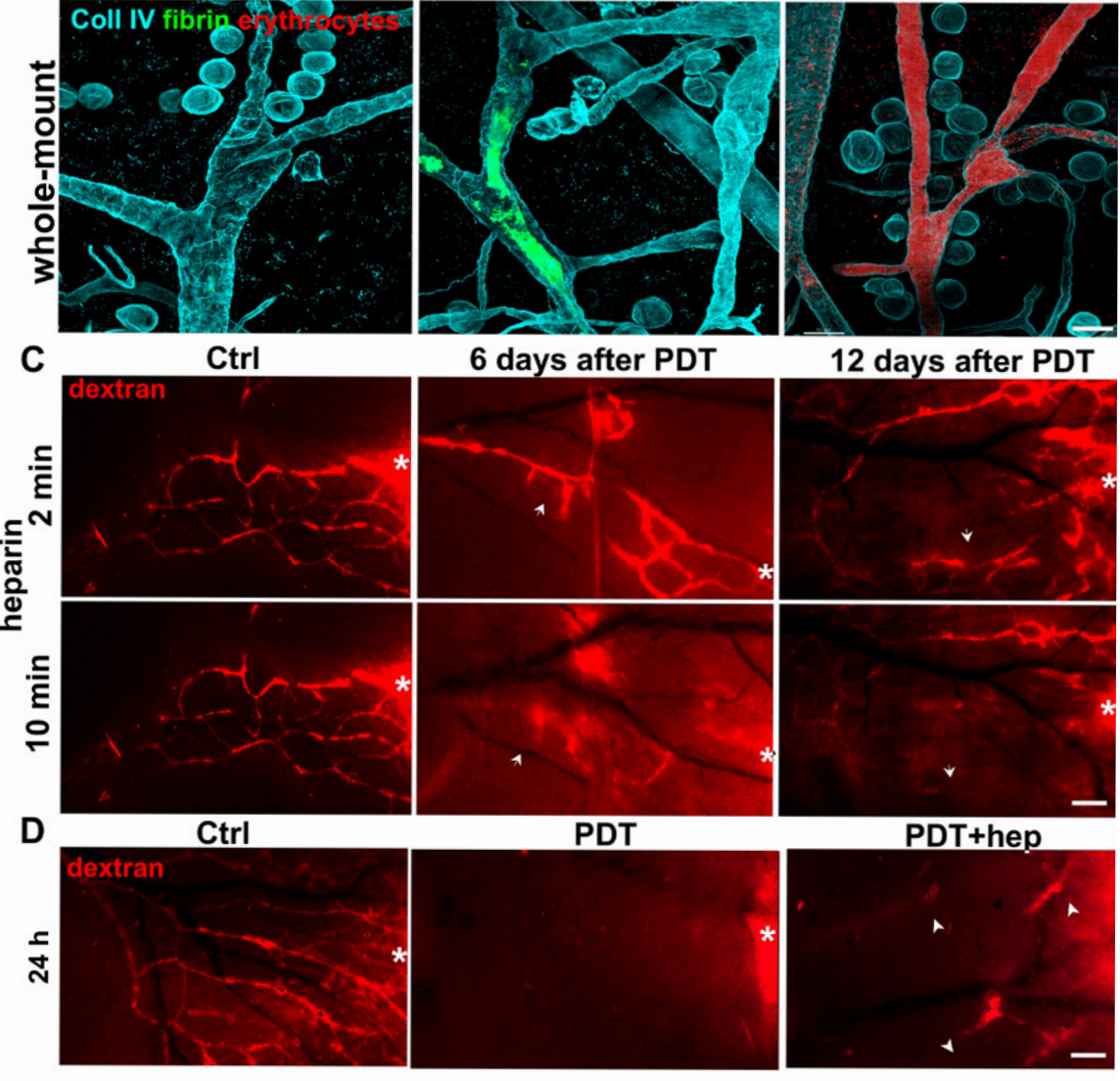

Ctrl





is fibrin clotting and not leukocyte accumulation within decellularized lymphatic directly followed endothelial cell death and was accurately correlated with lymphatic Decellularization of collectors leaves an intact tube of
occlusion. Interestingly, the antigen presenting cells (Fig. basementmembranethatisinitiallyfilled withimpermeable $\mathbf{8 B}-\mathrm{C}$ ) and $\mathrm{T}$ cells consist of the vast majority of cells that fibrin infiltrated ghost vessels while Gr1-positive neutrophils like matrix (Fig. 6). Since these ghost vessels were capable or monocytes and F4/80-positive macrophages and for at least short time drainage after decellularization, we monocytes only populated ghost vessels-surrounding asked whether lymphatic endothelium is at all needed for dermis (Fig. 8C). Antigen-presenting cells that populated fluid drainage and traffic leukocytes to the lymph nodes. ghost vessels expressed CD11c (Supplementary Fig. 4), To do that, we tried to block lymph clotting by intradermal
Figure 9. Heparin sustains lymphatic drainage in the deendothelized vessels. With ghost vessels drain antigens and immune of experimental design. Intradermal (i.d.). foction of Visudyne ${ }^{\ominus}$ mixed with 0.5 if
of heparin or saline is followed by collector maging (verteporfin lymphangiography). fibrinogen was injected intravenously (i.v.). same location in the ear skin was re-injected i.d. with $0.5 \mathrm{IU}$ of heparin in $2.5 \mu \mathrm{l}$ saline or saline the ear were imaged. Before fix-perfusion at indicated time point functionality of ear mphangiography using intradermal dextran
jection. B. Live imaging (live), Top: Heparin administration prevented intralymphatic clot Bottom, lymphangiography: In the absence ymphatics were able to drain fluorescent dextran. However, ghost vessels were
permeable and leaked the tracer into the dermis. Whole-mount imaging (fixed). Dece particles, demonstrated here with
large
autofluorescent erythrocytes. By repeating autofluorescent erythrocytes. By repeating
injections of heparin, erythrocytes from noncoagulated blood enter the non-occluded, of two consecutive doses of heparin on the day
of lymphatic decellularization was sufficient to maintain ghost vessel patency and drainage injection. Their leaky state persisted for 12 days. Arrows point to leaky lymphatic vessels.
D. Top: Lymphatic function was assayed with fluorescence lymphangiography before injection of EGFP-splenocytes into the ear
skin. Functional, PDT-decellularized Iymphatic collectors remain patent with concomitan
heparin injections but leaky. Bottom: Representative flow cytometry plots of EGFP
splenocytes in the draining lymph nodes (DLN) and non-draining lymph nodes (NDLN) in 24 hours after i.d. injection. Summary plot of flow cytometry EGFP+ cell counts. The red
triangles in PDT-heparin group correspond to ears where heparin injection preserved dextran lymphangiography done prior splenocytes were normalized to signal level of interstitia cells positive for these receptors. Scale bar he marker of dendritic cells $s^{53}$. (a) cessary for fluid drainage and cell trafficking ecess with a $692 \mathrm{~nm}$ laser (photodynamic therapy
PDT). Thirty min later fluorescently labeled 


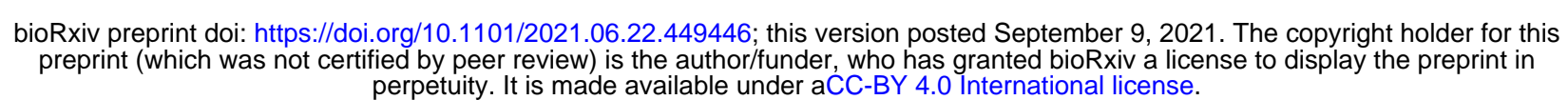

injection of heparin. This procedure was successful in 25\% cases, as determined by the absence of fluorescence fibrin deposits (Fig. 9B, live). Without intravascular lymph clots decellularized lymphatics were able to drain dextran however, their drainage was dysfunctional as they leaked not efficiently, lymphatics in the absence of endotial lining are capable of delivering macromolecules (e.g. antigens) to the draining lymph node. Furthermore, in the absence of endothelial lining and intralymphatic clots, remote segments of ghost vessels could be filled with erythrocytes, large particles that in contrast to fluid did not leak outside the basement membrane tube even 48 hours after injection (Fig. 9B, fixed). This indicates that endothelium is not necessary for cell passage to the draining lymph node. Interestingly, once ghost vessels were protected from the occlusion for 24 hours after the PDT, they stayed patent. Their draining state could be verified with lymphangiography even 12 days after PDT when all patent ly wat recover edothel lining were not perte to flurescent maker aleady few wintes after the inecton (Fig. 9C). Most importantly decellularized collocts whenever whever they remained patent and drained fluorescent dextan we also capable of trafficking leukocytes, most importantly dendritic cells, to the draining lymph node (Hg. 9D), proving that presence of endothelial lining is not necessary for this process. At the same time, these results prove that occlusion of collectors with clotted lymph is sufficient to completely block the drainage of macromolecules (e.g., antigens) and trafficking of any kind of cells from the tissue to the draining lymph node. Additionally, skin edema that develops one day after PDT was resolved on day 5 before any functional regeneration of lymphatics took place (Supplementary Fig. \%). This is however in agreement with early observations, wherein the absence of persistent inflammation lymphatic occlusion alone does not produce lymphedema $\mathrm{a}^{54}$.

Lymphatic occlusion is sufficient to hide allograft from the detection of the immune system

Due to the potent and acute cytotoxic response and little effect of the immunosuppression on allograft tolerance, skin transplantations confined to autografting ${ }^{55}$. However, cutaneous immunity has one significant vulnerability as its responsiveness depends on functional lymphatic system that connects the drained skin with secondary lymphoid $\operatorname{organ}^{57}$. Allografts are accepted indefinitely when transplanted to a location that could not drain to the lymph nodes because of induction of immune ignorance rather than tolerance ${ }^{57,58}$. Since we understood the occlusion mechanism and the kinetics of lymphatic regeneration, next, we planned to test the efficacy of the temporal lymphatic blockage on the prolongation of subcutaneous allograft acceptance. To do that we established highly reproducible and sensitive subcutaneous heart transplant mode ${ }^{59}$. In contrast to skin-over-skin transplant assays, transplantation of self-contracting cardiac tissue provides a natura rejection parameter tha can be visully assayed daily. Additionally, a pocket formed between cartilage 13 days cyclosporine-treated group ${ }^{62}$


C
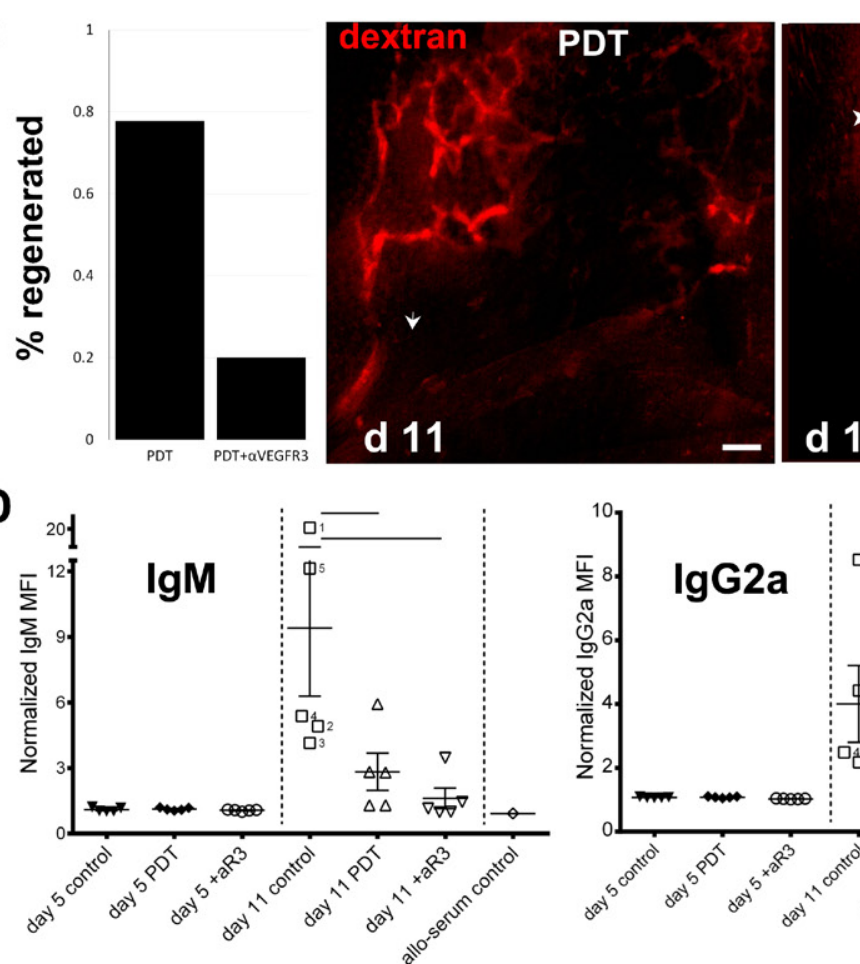

PDT aVEGFR3


E urvival was extended to 12 days when allografted hearts were implanted under PDT-treated skin. In addition, the rejection kinetics in control and PDT groups were different. While the control tissues were rejected across the 5 day period, the rejection in PDT group is almost binomina and occurred within one day. This could be explained by the high level of lymphatic regeneration homogeneity (Fig. 10C) but also the fact that ghost vessels before the regeneration of lymphatics become tertiary lymphoid organs ${ }^{\circ}$, with an accumulation of antigen presenting cells ready to be delivered to the draining lymph node Fig. 8). Conceivably, as the time of vessels regeneration was delayed with anti-VEGFR3 therapy (Fig. 10C), the time of allograft acceptance was almost doubled from the original 6 to 15 days. Interestingly, this value is better than presented with the same assay results on the effect of commercial cyclospoine (Sandimmune) teatment allograft rejection that occurred after 9 days in control and

Alloantigen-specific antibody that develops after the tissue implantation is generally considered not relevant for an acute cytotoxic response. Yet, their level and subtype constitute significant parameter in probing immune ignorance as the production of allo-specific antibody,

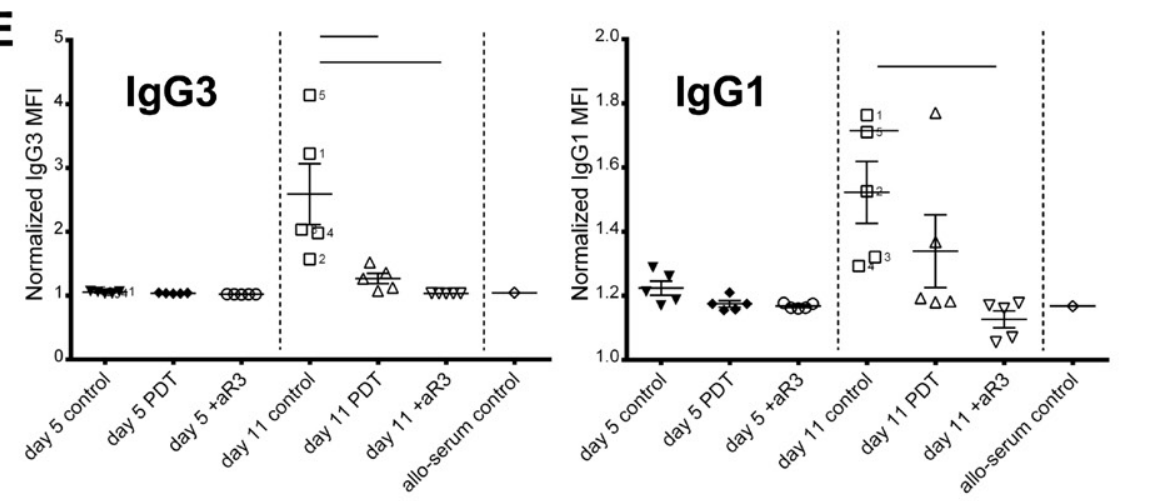

Figure 10. Lymphatic occlusion hides antigens and prevent rejection of skin allograft. Murine embryonic contractile cardiac fissue was transplanted under ear dermis of the fully immunocompetent mouse with or without lymphatic-specific photodynami A, B). A. Isografts from control group ( $n=6,5$ censored) and PDT group ( $n=6,6$ censored) continued contracting until the planne termination of the experiment (31 days) where lymphatic collector decellularization with PDT had no effect on isograft acceptance. (Mantel-Cox log-rank test ( $p<0.32$ ). All implanted hear fragments resume contraction after implantation. B. The median surviva mplanted under immuno-priviliged ear dermis with PDT-occluded lymphatics, the kinetics of allograft rejection was uniform, and graft median acceptance was prolonged to 12 days ( $n=18$ mice, 14 rejected, 4 censored). This median acceptance time of th allogeneic transplant grafted under PDT-decellularized lymphatics was prolonged to 15 days (Mantel-Cox log-rank test ( $p<0.0001$ ) with adjuvant anti-lymphangiogenic therapy with intraperitoneal administration of anti-VEGFR3 antibody (PDT aVEGFR3 group first 5 mice, 10 rejected, 1 censored). All censored cases in allograft groups were due to failure to resume contraction during the ymphatic drain 11 days ater treatment in PDT roup In contrast mice reciving adjuVant QVEGFR3 antibodies after PDT ymphatic drainage 11 days after treatment in PDT group. In contrast, mice receiving adjuvant $\alpha$ VEGFR3 antibodies after PDT
induced lymphatic decellularization had only 2 out of 10 ears that resumed drainage ( $p=0.023$, Fisher exact test). Representative 

control is shown as a point of reference. Bars indicate the difference between groups tested with ANOVA and Holm-Sidak post-
priori test at $\mathrm{p}<0.05$. and antibody class switching reflects the availability of therapeutic relevance is limited to extemporary, e.g., alloantigens in the lymph node $e^{63}$. The seven-fold relative inhibition of bacterial spread in early acute inflammation, increase of the earliest released IgM alloantibody was the as previously reported ${ }^{64-66}$. Similar to thrombomodulin highest between all tested isotypes (Fig. 10C) indicating inhibition, decellularization of lymphatic intima lead to that anti-lymphatic (PDT) therapy reduced the increase massive and continuous lymphatic occlusion independent of allospecific antibody to 1.4 fold (2.4-times), with on LEC expression profile or their location within collector further reduction to the basal level ( 0.4 fold increase) leaving 'ghost vessels' composed of acellular basemen was measured when anti-lymphatic therapy (PDT) was membrane filled with lymph clots (Fig. 5-6). Experiments followed by lymphatic regeneration delay with aVEGFR3 with injection of necrotizing compounds, i.e. TritonX therapy. Even though the level of antibody in PDT and and $\mathrm{FeCl}_{3}$ (Fig. 5) aimed to confirm previous observation PDT- $\alpha$ VEGFR3-treated groups were not different to the where intradermally administrated turpentine or $\mathrm{FeCl}^{64,66}$ respective basal levels, the pattern of antibody decline lead to cessation of lymphatic drainage by intralymphatic (renge by intralymphatic with middle values after only PDT and almost basal after PDT $+a$ VEGR 3 was reproduced for every subtype (five curves not different, $\mathrm{p}<0.05)$. This means that rather
than the development of immune tolerance, the immune reaction was only delayed and that alloantigens that remain outside the reach of the lymph node did not lead to T-cell tolerance. Instead, these alloantigens are 'ignored',
and the alloreactive T cells remain functionally intact and can be engaged when tissue clearance is restored.

\section{Discussion}

Here we characterize anti-clotting properties of the lymphatic collecting vessels and their application in the on-demand cessation of antigen drainage and trafficking of antigen presenting cell. This leads to the creation of subcutaneous immune-privileged zones where allografts can be protected from immune-recognition without immunosuppression. We show that fluid drainage and immune cell trafficking entirely depend on lymphatic vessel ability to sustain lymph liquidity.

Physiological function-mechanisms of intralymphatic clotting

Regardless of the method of lymph clotting induction intravascular lymph clots interfered with essential lymphatic functions, fluid and macromolecule evacuation from the drained area of the skin. Intralymphatic clots were detected by copolymerization of fluorescently were detected by copolymerization of fluorescently labeled fibrinogen. Because FITC-fibrinogen was injected intravenously, first it had to leak with other plasma proteins Later these proteins were collected by lymphatics and drained as naturally formed lymph. Therefore the fluorescent labeling of intralymphatic clots reflected the natural process of lymph coagulation.

In contrast to selective induction of pro-hemostatic $\mathrm{T}$ expression that resulted in discrete and sparse lymp clots deposition within collecting lymphatics (Fig. 1-2), inhibition of constitutively expressed thrombomodulin resulted in widespread lymph clotting inside efferen vessels (Fig. 3B and 4B,C). These lymph clots formed within intact lymphatic intima were cleared within subsequent 16 hours, therefore, their physiological or

\section{reviewed by Drinker and Field ${ }^{14}$ ).}

Both, TritonX and ferric chloride exerted toxicity at the site of injection but also completely blocked the skin drainage and induced lymph clotting in the lymphatics ocated remotely to the site of intradermal injection. The same effect of lymphatic occlusion but without advers skin, necrosis was achieved with PDT-induced lymphatic decellularization (Fig. 6-10). Due to the high specificity of anti-lymphatic treatment and the long-lasting (9 days) drainage occlusion, we used this method to study the effects of intravascular lymph clotting on lymphatic physiology and local immune responses.

Despite the type of lymphatic treatment, lymph clotting was an endpoint that preceded interference or complete blockage of fluid drainage. Even though leukocytes involvement in fibrin polymerization and maturation within lymphatics could not be excluded; the initial ymph coagulation occurred before leukocytes infiltratio Fig. 2 and 8). Therefore, in one way or another lymph clotting was dependent on lymphatic endothelium that either expressed TF after stimulation lost anti-hemostatic properties after thrombomodulin blocking or was destroyed by detergent lysis (Triton-X-100), exposure to free-radicals generated by $\mathrm{FeCl}_{3}$ or free-radicals generated by light-activated verteporfin (i.e., lymphatic-specific PDT). However the duration of lymphatic occlusion varies significantly between the metabolic and noxious methods of lymph clotting from less then 22 hours with preserved intact ( when collectors were dying RNA and polyphosphates that can activate focly charged RNA and polyphosphates that can activate factor XII of the contact-dependent (intrinsic) coagulation pathway ${ }^{45}$. Intrinsic pathway can be also continuously activated by the constant exposure of lymph to the unmasked basement membrane collagens ( ${ }^{33}$ and Fig. 6C-D), which in the absence of endothelial membrane-bound antithrombotic thrombomodulin( ${ }^{19}$ and Fig. 1A) can lead to recurren activation of lymph coagulation even when the initial clo is cleaved 4-5 days after PDT-driven decellularization of lymphatics (Fig. 6F). Stability of intralymphatic clots is additionally enhanced by the loss of the endothelium that is a source of tissue plasminogen activators ${ }^{21}$, whic production is enhanced after pro-thrombotic stimulation of endothelium (Fig. 3D). Therefore, restoration of lymphatic intima should be the critical factor in stable preservation of collector patency. This conclusion is in agreement with our subsequent finding that lymph clotting but not the loss of endothelial lining was a direct cause for lymphatic occlusion to fluid and cells. This is indicated by a delayed block of drainage that occurred hours after endothelial death (Fig. 7), and most notably by sustained fluid drainage and cell trafficking through PDT-decellularized collectors when fibrin deposition was inhibited with heparin (Fig. 9). Even though decellularized collecting lymphatics were leaky to drained dextran, this experiments indicated that endothelial lining is not indispensable for the most affirmed functions of lymphatics, the fluid drainge, and cell trafficking and instead lymphatic endothelium is primarily needed to support collector's patency.

Intravascular lymph clots delay of allograft rejection -therapeutic applications

The lack of conventional lymphatic drainage is a characteristic feature shared between passive immune ignorance $^{57}$ and immune privilege at sites like placenta, cheek pouch, cornea or central nervous system $^{67-69}$. Furthermore, it was shown that surgical separation of draining lymphatics blinds the draining lymph nodes to antigens residing within the blocked area and prevent the development of adaptive immune response against allograft at least for the time alternative lymphatic routes allograft at least for the time alternative lymphatic routes
developed $^{70,71}$. Due to extensive and traumatic surgery, these experiments lack translational potential. Instead, they provided a proof of concept where elimination of antigen drainage path leads to the formation of immune privilege zone where foreign antigens remain ignored by adaptive immunity. Long-lasting occlusion of lymphatic collector that follows specific eradication of lymphatic intima allowed us to mimic the effect of mechanical laceration of the skin and prove the therapeutic applicability of the antihemostatic properties of lymphatic endothelium. Instead hemostatic properties of lymphatic endothelium. Instead of skin transplant, where rejection is defined by subjective
parameters $^{72}$, we used sensitive cardiac graft where the parameters", we used senstive is defined immune rejection is manifested by the loss of spontaneous contractile function . Indeed, the rejection of allografts but also the development of allo-specific antibodies was delayed after PDT-dependent decellularization of collectors and correlated with drainage recovery from the skin surrounding the graft (Fig. 10). Importantly, the time of graft acceptance was doubled as the immune response was delayed when lymphatic regeneration was inhibited with VEGFR3 blocking antibodies. Possibility to further prolong allograft hiding from the immune-recognition with anti-lymphangiogenic therapy indicates that there are still opportunities for further enhancements in the adjuvant therapies, e by reinforcing clotted lymph with matrix-boup combined relatively non-invasive treatments, lymphatic ellularization with PDT and subsequent inhibition of (delay by $47 \%$ ) than . me in a form of clinicallymun (delay by $30 \%$ ), when tested in cartiac allograft model ${ }^{62}$. However, it is important to appreciate the difference between these treatments, while lymphatic occlusion leads to immunological ignorance by hiding tissue from immune recognition in draining lymphatics, cyclosporine suppresses already developing an immune response.

Anti-clotting function of lymphatics in health and disease We described the overlooked function of lymphatic vessels that might have significant consequences in various pathologies and therapies that until now could not be adequately appreciated. In homeostasis anti-hemostatic mechanisms helps sustain lymph as a liquid but during the acute inflammation, the protein-rich exudate that leaves blood vessels does not enter lymphatics, helping to circumscribe the infection from draining to the efferent vessels and eventually bloodstream, reviewed by Menkin ${ }^{74}$ and Drinker ${ }^{14}$. This phenomenon of 'fixation' of bacteria, protein, and particles to the site of early inflammation were attributed to the mechanical obstruction of lymphatics with fibrin leaking from inflamed or injured blood vessels. Unfortunately, as it could explain, e.g. why acute lymphangitis, an infection of lymphatic collectors is predominantly caused by plasmin-activating positive group A streptococci bacteri ${ }^{75}$ the research on in situ group A streptococci bacteria ${ }^{35}$ the research on in situ the other hand, ablity of dysfunction of lymphatics to sustain nonclotted lymph might contribute to their pathologies with diopathic or confound origin. Surprisingly, even in case of thoroughly studied lymphedema, all causative factors are not known as lymphatic occlusion is a necessary but still insufficient factor to induce sustained lymphedema in nimal models ${ }^{22,76,77}$. Also, lymph clots within lymphatics around the granuloma that form after worm death during the pathogenesis of filarial lymphedema ${ }^{24}$ intuitively carry a notion that lymph clotting and inflammation might be important priming factors of the disease. Filaria-induced lymphedema develops after repeated instances of worm deaths and bacterial lymphangitis that additionally lead to lymphatic endothelium injury and fbrotic renly lead f lymphatic collectors ${ }^{78}$. Along these lines, our results collocto clusion is tan

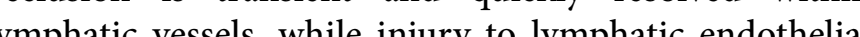
lining is sufficit for prolonging occlusion of the But even after PDT-ind But even after PDT-induced lymphatic decellularization, ghost vessel eventually regenerated instead of becoming fibrotic. PDI-decellularization is a specific and sterile anti-lymphatic treatment while trauma combined ith infectious inflammation might produce longvessels occlusion with collagenous fibrosis, elements of which we could observe in lymphatics regenerating lymphatics should be the primary parameter that requires 


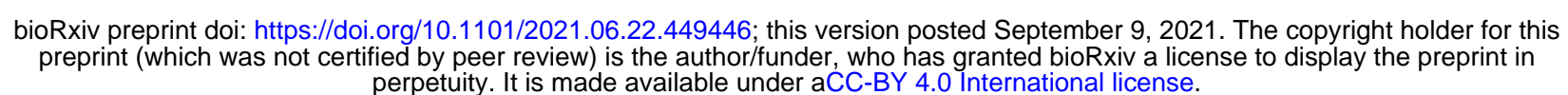

consideration before designing lymphatic implants, e.g., artificial collectors lympho-venous shunts or lymphatic collateral neovessels ${ }^{79}$ as they must anchor functional antihemostatic mechanism in order to canalize the edematous hemostatic mechanism in order to canalize the edematous property of lym hatic has not beew yet filly apprecta property of lymphatic has not been yet fully appreciated it is possible that lymph clotting and subsequent fibrosis is an underestimated complication unwittingly hampering lymphedema treatment. For example, unpredictable clotting and fibrinolysis cycles could explain the fact tha most silicon tubes implanted in lymphedema patients did not drain the fluid along their lumen even though it did nor undergo fibrosis and remained paten $\mathrm{t}^{8 \mathrm{0}}$.

The active regulation of lymphatic drainage might also affect tumor metastasis. Entrapped tumors or associated cells might activate fibrinolytic mechanisms and use within the lymphatic system. This scenario is suppotion winin the lym sactic system. This sce is supported by experinnts whe depletion of fibrinogen inhibits lymphatic netastasis but has no effect on primary tumor growth $^{\text {bi }}$. Contrarily, completely occluded lymphatics might stop the spread of metastatic cells, likely the mechanism of PDT treatment that blocked the metastasis spread via peri-tumoral lymphatics ${ }^{82}$. In this situation lymphatics, control over lymph hemostasis is a potential new avenue for the research that could, e.g. aim at reinforcement of fibrin or local inhibition of fibrinolysis collectors. Strengthening of intralymphatic clot resilience could also be used to delay further the regeneration of lymphatics occluded with PDT and by this extend the time subcutaneous allografts are masked and ignored by the immune system. Accessibility of the dermal lymphatics and low-risk subcutaneous surgery might permit therapeutic low-risk subcutaneous surgery might permit therapeutic
prosthetic transplantation of small hormone-producing various tissue fragments or cells, like Langerhans islets Here we identified anti-coagulatory properties of lymphatic collecting vessels and demonstrated their permissive influence on essential lymphatic functions, i.e., fluid drainage and cell trafficking. Interference with these properties lead to a various level of lymphatics

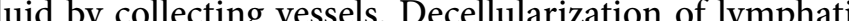
vessels resulted in complete loss of anti-coagulatic


(biclusion. phenotype of lymphatic vessels, as well as their drainage could be rescued with the injection of heparin, indicating a fundamental impact of lymphatic anti-clotting a fundamental impact of lymphatic anti-clotting properties on their primary function. Finally, we showed
that intralymphatic occlusion correlated with the delay that intralymphatic occlusion correlated with the delay mmune response, the potential alternative approach to chemical immunosuppression after allogeneic tissue transplantation.

\section{Methods}

Animal experiments

Animal experiments were performed at the EPFL (Lausanne, Switzerland), the University of Chicago and the Medical University of Warsaw (tail edema experiments). Procedures performed on animals were in strict accordance with the Swiss Animal Protection Act the ordinance on animal protection and the ordinance on animal experimentation. We confirm that our Institutional Animal Care and Use Committee (IACUC) named Commission de Surveillance de l'Etat de Vaud (Permit Number: 2646 and 2687), specifically approved this study or in accordance with protocols approved by the Institutional Animal Care and Use Committee at EPFL (permit number 72414). Procedures performed at the University of Chicago were performed according to rules and protocols at the University of Chicago were performed according to the guidelines approved by the Ethics Committee of the Medical University of Warsaw. $\mathrm{BALB} / \mathrm{c}$ female or $\mathrm{C} 57$ mice were purchased from Jackson aboratories or Charles River, maintained and breed in a pecific pathogen-free barrier facility and used between 6 to 10 weeks of age unless stated otherwise.

\section{Anesthesia and hair removal}

Three days before each experiment, ears were treated with depilation cream (Veet ${ }^{\oplus}$ Hair Removal Cream, Slough, UK) for $10 \mathrm{~s}$ followed by thorough rinsing with water. For PDT, mice were anesthetized with $2.5 \%$ isofluorane (Rothacher GmbH, Bern, Switzerland) and kept under 1.5 $\%$ isoflurane for the duration of the experiment. Mouse core temperature was maintained at $37^{\circ} \mathrm{C}$ throughout the experiment (DC Temperature Control System, FHC Inc., Bowdoin, MA). All intradermal injection in the dorsal mouse ear $(0.5 \mu \mathrm{ll})$ was done using a microsyringe (Hamilton, Reno, NV, USA) equipped with $33 \mathrm{G}$ needle.

\section{(PDT) on the mouse ear}

Mouse head was epilated under isoflurane anesthesia 24 $\mathrm{h}$ before the procedure. Ears were treated with epilation cream (Veet) briefly for $10 \mathrm{~s}$ at the end of the head epilation process, and mouse head was washed with plenty of water. The mouse was allowed to recover in a cage with food and water ad libitum. On the day of the experiment, a mouse was anesthetized with $2.5 \%$ isoflurane and kept unde $1.5 \%$ of isoflurane for the duration of the experiment. $0.5 \mu \mathrm{l}$ verteporfin in the form of liposomes (Visudyne ${ }^{\circledR}$ Novartis Ophthalmics, Hettlingen, Switzerland) freshly reconstituted from the powder as previously described was injected in the top of the ear with Hamlton syinge (Reno). Injection site of the ear was cover by a syinge (Reno). Injection site of the ear was covered by aluminum foil and 1 min later, the ear was irradiated with nonthermal laser light (Biolitec) or 2W LED laser (Modulight) at $689 \pm 2 \mathrm{~nm}$ delivered through an optical fiber containing a frontal light distributor with a lens (Medlight) with the irradiance of $50 \mathrm{~mW} / \mathrm{cm} 2$, and light dose of $100 \mathrm{~J} / \mathrm{cm} 2$. Light doses were adjusted with neutral density filters and measured with a calibrated Field-Master GS power meter (Coherent).
Decellularization of lymphatics with detergent or $\mathrm{FeCl}$ $5 \%$ Triton-X-100 (TX100) and $\mathrm{FeCl}_{3}$ (Ferrum chloride) were dissolved in water and filtrated through the $0.22 \mu \mathrm{m}$ membrane. $0.5 \mu \mathrm{l}$ of TX100 or Ferrum chloride was injected in two adjacent spots at the top of the ear dorsal dermis and immediately after that FITC labeled fibrinogen was injected i.v.

\section{In vivo labeling of fibrin}

$40 \mathrm{ml} 10 \mathrm{mg} / \mathrm{ml}$ fibrinogen (SIGMA) dissolved in in PBS was reacted with $1.6 \mathrm{ml}$ of $4 \mathrm{mg} / \mathrm{ml} \mathrm{FITC} \mathrm{(ThermoFisher)}$ for 1 hour and fibrinogen was dialyzed 4 times to $4 \mathrm{~L}$ each time of PBS. After that fluorescent fibrinogen was aliquoted and stored at $-80^{\circ} \mathrm{C}$ until used. After thawing $200 \mu \mathrm{l}$ of labeled fibrinogen was injected iv into mouse tail vain up to 15 to 30 minutes after treatment thouse toll vain up to 15 to 30 minutes a nfer treat. into mouse coagulation (PDT, thrombin, cytokine injection).

Blocking thrombomodulin and TF expression on lymphatics $1 \mathrm{U} / \mu \mathrm{l}$ thrombin was reacted with $1 \mathrm{mM}$ phenylmethylsulfonyl fluoride (PMSF, Sigma) for 2 hours and residual PMSF was removed on the Zeba ${ }^{\mathrm{TM}}$ desalting column (ThermoFisher Scientific). This treatment ralting $85 \%$ of thrombin inactive as determined with the delayed fibrin clotting (from 45 seconds to 270 seconds).

Complete inactivation of thrombin was done by reacting of $50 \mu \mathrm{l}$ of $1 \mathrm{U} / \mu \mathrm{l}$ thrombin with $1 \mathrm{mM}$ $p$-amidinophenylmethylsulfonyl fluoride (p-APMSF, EMD Millipore Corp. ${ }^{43}$ ) $\mu \mathrm{l}$ of thrombin for 2 hours and residual PMSF was removed on the Zeba ${ }^{\mathrm{TM}}$ desalting column. $0.5 \mu \mathrm{l}$ of inactive thrombin or partially inactivated thrombin was injected intradermally alone or with 10ng/ $\mu$ TNFa or $10 \mathrm{vg} / \mu \mathrm{l} \mathrm{II-1} \beta$ in the ear top dorsal dermis.

\section{Splenocytes trafficking into lymphatics}

splenocytes from BALB/c mouse expressing eGFP under ubiquitin promotor were isolated from the mouse spleen by grounding the spleen through the strained with $70 \mu \mathrm{m}$ pores. Red blood cells were lysed with RBC lysing solution $150 \mathrm{mM} \mathrm{NH} \mathrm{Cl}$ and $10 \mathrm{mM} \mathrm{NaHCO}_{3}$ for 5 minutes, (150 $\mathrm{mM}_{4} \mathrm{Cl}$ and 10 mM Nated washed twice 25 mM HEPES, PH 7.5. Cells were spun buffered with 25 mM HERS, pH 7.5. Cells were spun down for the last time, loaded to the Hamilton syringe with the pipette tip. After mounting $33 \mathrm{G}$ needle, 2.5 $\mu l$ slurry was injected into the same location of the ear where verteporfin and heparin or Ringer was injected on the previous day.

\section{Fluorescent intravital lymphangiography}

Two hours after PDT and every 2-3 days $1 \mu \mathrm{l}$ of TRITC dextran $(10 \mu \mathrm{g} / \mathrm{ml})$ was injected in the location of initia verteporfin injection. Draining lymphatics were imaged with stereomicroscope after the ear was placed between two glass slides. For intravital lymphangiography, ventral skin with underlying cartilage was separated and removed from dorsal, PDT-treated skin and primary rabbit, anticollagen IV antibody, was applied for 20 minutes. After brie washing with Ringer's buffer, secondary streptavidin-647 was applied for 5 minutes; tissue was washed and imaged ing automated fluorescent stereomicroscope (M205 FA, Lica Microsystems) coupled to the sensitive color camera DFC350 FX (Leica Microsystems). The following objectives have been used: Plan Apo 1x, NA 0.035; W.D. 60 or Plan Apo 2x, NA 0.07: W.D. 60 (Leica Microsystems). Intracardiac perfusion and whole-mount staining of a mouse ear

Mice were sacrificed with $\mathrm{CO} 2$ and exsanguinated by intracardiac perfusion with $20 \mathrm{ml}$ Ringer's buffer and 25 mM HEPES; pH 7.5, 330 mOsM) at a constant gravitational pressure of $120 \mathrm{~mm} \mathrm{Hg}$. Ringer's buffer was then changed to osmolarity-corrected zinc fixative ( $\mathrm{Zn}$ fix) solution [26] $(4.5 \mathrm{mM} \mathrm{CaCl}, 52 \mathrm{mM} \mathrm{ZnCl} 2,32 \mathrm{mM}$ $\mathrm{Zn}(\mathrm{CF} 3 \mathrm{COO}) 2,2 \mathrm{mM}$ Triss, $38 \mathrm{mM}$ glycine; $\mathrm{pH} 6.5,340$ $\mathrm{mOsm} / \mathrm{L}$ ) and ears were cut and placed in ice-cold $\mathrm{Zn}$ fix with $1 \%$ Triton $x-100$ for at least $24 \mathrm{~h}$. After that, the ventral part of the skin was removed together with cartilage and muscles, and dorsal dermis was washed in TBS $(140 \mathrm{mM}$ $\mathrm{NaCl}, 25 \mathrm{mM}$ Triss, $\mathrm{pH} 7.5$ ) for $6 \mathrm{~h}$, blocked with $0.5 \%$ casein TBS (blocking solution) for $2 \mathrm{~h}$, and incubated with antVE-cadherin, anti-Lyve-1, and biotinylated anti-collagen type IV antibodies $(10 \mu \mathrm{g} / \mathrm{ml})$ in blocking buffer for 24 h. After washing in TBS with $0.1 \%$ Tween 20, tissue was incubated with the appropriate secondary antibody (10 $\mu \mathrm{g} / \mathrm{ml}$ ) for another $24 \mathrm{~h}$, washed in TBS and dehydrated with $70 \%$ and $100 \%$ ethanol before it was cleared with $2: 1$ benzyl benzoate/benzyl alcohol solution supplemented with $25 \mathrm{mg} / \mathrm{ml}$ propyl gallate (refractive index 1.56). The tissue was mounted on a glass slide and imaged using $\mathrm{HC}$ PL APO 20x, NA 0.70 or HCX PL APO 63x, NA 1.40 lenses of a Leica SP5 confocal microscope (Leica). Stacks of images were analyzed with Imaris 7.4 (Bitplane AG).

\section{Transplant experiments}

PDT was performed on ears on day 0 and on the next day newborn (1-2 days after birth) mice hearts from allogenic or syngeneic mice were cut in half and incubated in DMEM media. The incision was done on the ventral side of the mouse ear and spatula was inserted through the cartilage to the end of the dorsal, separating cartilage from dorsal ear skin. Inside the forming chamber, halve ear was inserted, and the ventral incision was sealed with surgical glue. $50 \mu \mathrm{l}$ of the blood was collected on day 5 and 10 , and isolated serum was frozen for further analysis. Heartbeat in mouse ear was monitored daily after shor isoflurane anesthesia. Until the beating stopped when mice were perfused, and their ears were zinc-fixed for further analysis.

Detection of allo-specific IgM and IgG in grafted mice Thymocytes from the allogenic background were isolated from 4 weeks old mice at $10 \times 10^{6} / \mathrm{ml}$, and $50 \mu \mathrm{l}$ of cells were mixed with $5 \mu$ l of mouse serum isolated from mice bearing transplants on day 5 and 10. After washing, cells were incubated with appropriate isotype antibody or antiIgM antibody, re-suspended for FACS buffer for acquisition in Beckman Coulter CyAn ADP flow cytometer. 


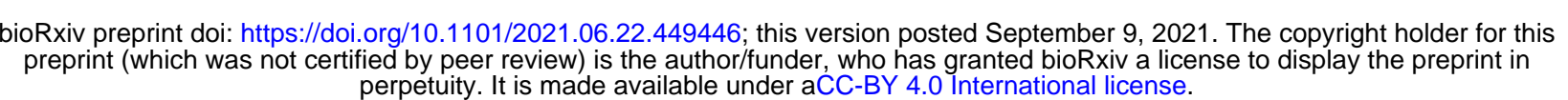

\section{Acknowledgement}

The authors are grateful to S. Hirosue for editing the manuscript and advice, V. Triacca, E. Guc and J. Kilarska for their technical assistance and advice. The authors are grateful to the EPFL Bioimaging and Optics Platform and Histology Core facility for their technical assistance. Funding for this project was provided in part from the European Commission Framework Project 7 Angioscaff, and from the European Research Commission Advanced Grant LymphImmune, 323053 and Polish-Swiss Research Programme (PSPB-057/2010 to JG, WWK and MAS).

\section{References} Rusznyák, \&al. (1967). Lymphatics and lymph circulation. Physiology
Dis and pathology, Pergamon Press \& Wilkins co
Miller, $a$ ( Miller, \&al. (2000).

lymph. Thromb Haemost flammation. $\mathrm{Br}$ Med Opie (19,3). Thrombosis and occlusion of lymphatics. J Med Res
Copley, \&al. (1942). Bleeding time, Iymph time, and clot resistance in



Mayanskii, \&all, (1966). A c cion in Iymph. J Physiol the blood and lymph. Bull Exp Biol Med
Le,\&al. (1998). Hemostatic factors in rabbit limb lymph: Relationship to mechanisms regulating extravascular coagulation. Am J Physid
Leak, \&al. (2004). Proteomic analysis of fymph. Proteomics

Lippi, \&al. (2012). Hemostatic properties of the lymph: Relationships

with occlusion and thrombosis. Semin Thromb Hemost
Brinkman, \&al. (1996). Vessel wall-mediated activation

coagulation system. Vascular control hemost. Harwood Academic
Pubisions Publishers.
Drinke, \&. (1933). Inflammation. Lymphatics, Ilymph and tissue fluid.
The Williams \& Wilkins Co.

Aukland,\&al. (1993). Interstitial-lymphatic mechanisms in the control

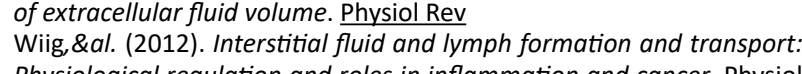

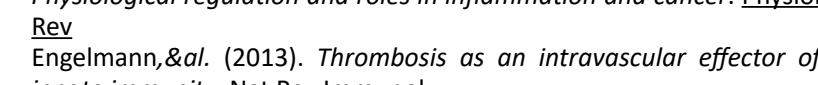
innate immunity. Nat Rev Immunol

in and its role in inflammation. Semir

Maruyama, \&al. (1985). Thrombomodulin is found on endothelium of arteries, veins, capillaries, and lymphatics,
of human placenta. J Cell Biol

Kirchofer, \&al. (1994). Endothelial cells stimulated with tumor necrosis factor-alpha express varying amounts of tissue factor resulting
in inhomogenous fibrin deposition in a native blood flow system. Effects of thrombin inhibititrs. J Clin Invest Laschinger, \&al. (1990). Production of plasminogen activator and
plasminogen activator inhibitor by bovine lymphatic endothelial cells:

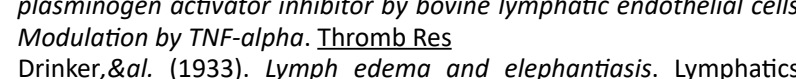

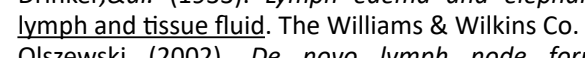

Olszewski (2002). De novo lymph node formation in chronic inflammation of the human leg. Ann N Y Acad So
Case, \&al. (1991). Vascular abnormalities in exp

Iyphatic filariasis. Lymphology Fader, \&al. (1986). Evolution of lymph thrombi in experimental brugia malayy infections: A scanning electron microscopic study. Lymphology
Hara, \&al. (2013). Lymphoedema caused by idiopathic lymphatic thrumbus. 0 Plast Reconstr Aesthet Surg

Forster \&al (2006). Tissue foctoedema treatment. Phlebologie

aspects. Clin Chim Acta
Ruf, \&al. (2011). Tissue factor and cell signalling in cancer progression
and thrombosis. IThromb Haemost and thrombosis. J J Thromb Haemost
Padera, \&al. (2002). Lymphatic mettos Idell (2003). Coagulation, fibrinolysis, and fibrin deposition in acute lung injury. Crit Care Med

us metastases of breast carcinoma. Rare valignant skintumors. Springer.
34. White-Adams,\&al. (2010). Laminin promotes coagulation and Haemost Bevilacqua, \&al. (1984). Interleukin 1 (IL-1) induces biosynthesis and
cell surface expression of procoagulant activity in human vascular endothelial cells. $\mathrm{J}$ Exp Med
Lupu, \&al. (2005) Tissuefor

up-regulated within arterial branching areagulation is preferentially

escherichia coli sepsis. Am J Pathol
37. Liu, \&al. (2004). Trombin and tumor necrosis factor alpho
synnergistically stimulate tissus factor expression in human endothelial cells: Regulation through c-fos and c-jun. $J$ Biol Chem

K. Kilarski, \&al. (2013). Intravital immunoffuorescence for visualizing the microcirculatory and immune microenvironments in the mouse ear van Hinsbergh (2012). Endothelium--role in regulation of coagulation and inflammation. Semin Immunopatho

(2005). Possive feedbacks of coagulation: Their role threshold regulation. Arterioscler Thromb Vasc Biol
Mackman (2009). The role of fissue factor and factor

ia in hemostasis. Fuesthes-Prior,\&al. (2000). Structural basis for the anticoagulant activity of the thrombin-thrombomodulin complex. Nature Laura, \&al. (1980). (p-amidinophenyl)methanesulfonyl fluoride, an irreversible inhibitor of serine proteases. Biochemistry
Chen, \&al. (2010). Sterile inflammation: Sensing and reacting to damage. Nat Rev Immunol

Esmon (2010). Far from the heart: Counteracting coagulation. Nat Med

Annu Rev Med (2008). Granulocytes do not express but acquire monocyte-derived tissue factor in whole blood: Evidence for a direct transfer. Blood

.

4. Kilarski, Qual. (2014). Optimization and regeneration kinetics of lymphatic-specific photodynamic therapy in the mouse dermis.
Angiogenesis

Angiogenesis Ini, \&al. (2004). Inhibition of vascular endothelial growth factor (VEGF) signaling in cancer causes loss of endothelial fenestrations, regression
of tumor vessels, and appearance of basement membrane ghosts. Am of tumor vessels, and appearance of basement membrane ghosts. Am
J Pathol

tgf-beta. Cell Tissue Res

of, tissue matrix cong-term intravital immunofluorescence imaging

Randolph, \&al. (2005). Dendritic-cell trafficking to lymph nodes throug

Drinker, \&al. (1933). Relation of the

consequences of lymphatic obstruction. Lymphatics tymph and the fluid. The Williams \& Wilkins Co.

gnition and rejection of allogeneic skin grafts. Immunotherapy
Lund, \&al. (2016). Lymphatic Lakkis,\&al. (2000). . Immunologic 'ignorance' of vascularized orga transplants in the absence of secondary lymphoid tissue. Nat Med
Yamagami, \&al. (2001). The critical roly of lymph nodes in corned alloimmunization and graff rejection. Invest Ophthalmol Vis Sci ear. Am J Anat McFarland, \&al. (2009). Skin allograft rejection. Curr Protoc Immunol
Ruddle (2014). Lymphatic vessels and tertiary lymphoid organs. J Clin Ruduest
Invest Gorecki, \&al. (1991). Evidence that liposome incorporation of
cyclosporine reduces its toxicity and potentiates its ability to prolong survival of cardiac allograffts in mice. Transplantation Rev Immunol Menkin (1931). Studies on inflammation : Vii. Fixation of bacteria and

of particulate matter at the site of inflammation. $\mathrm{J}$ Exp Med
Menkin (1931). Studies on inflammation : Vi. Fixation of trypan blue in inflamed areas of frogs. IExp Med

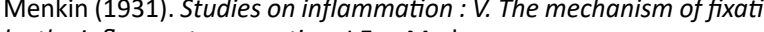
by the inflammatory reaction. $J$ Exp Med

$\frac{\text { circumscribe Med }}{\text { Medawar (1908) }}$ Stin hor (1948). Immunity to homologous grafted skin; the fate of to the anterior champer of the exe. Bry Exp Pathe Simpson (2006). A historical perspective on in

Aspelund,\&al (2015). A dual A dural lymphatic vascular system that drains
71. Barker, \&al. (1968). The role of afferent lymphatics in the rejection of skin homografts. JExp Med
Lambert, \&al. (1965). The role of the lymph trunks in the response to allogeneic skin transplants. Transplantation Joffre, \&al. (2008). Prevention of acute and chronic allograft rejection with cd4+cd25+foxp3+regulatory Lorentz, \&all. (2011). Engineered aprotinin for improved stability of

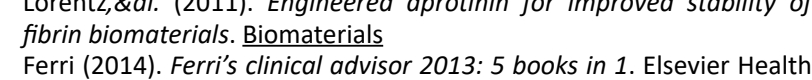
Sciences.

6. Rutkouski,\&al. (2006). Characterization of Iymphangiogenesis in a

77. Rutkowski,\&al. (2006). Secondary lymphedema in the mouse tall Lymphatic hyperplassia, VEGF-C upregulation, and the protective role of MMP-9. Microvasc Res

lymphatic remodeling and contractile dysfunction in filarial disease pathogenesis. Microcirculation
Patel, \&al. (2015). Lymphatic

作

breast cancer patient. Gland Surg

Olszewski, \&al. (2015). A novel method of edema fluid drainage in o osstructive lymphedema of limbs by implantation of hydropho


metastasis, but not primary tumor growth or angiogenesis,
diminished in fibrinogen-deficient mice. Cancer Res

82. Tammela \&al. (2011). Photodynamic ablation of lymphatic vessels and intralymphatic cancer cells prevents metatastasisis Sci Trans I Med
3. islet and cellular transplantation. Nat Biotechnol 


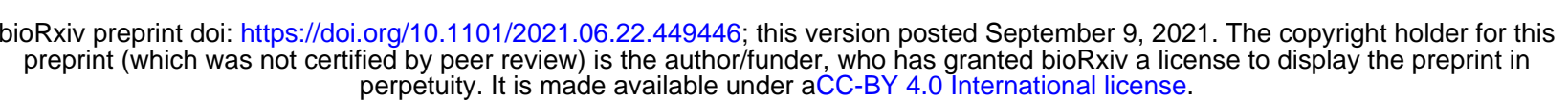

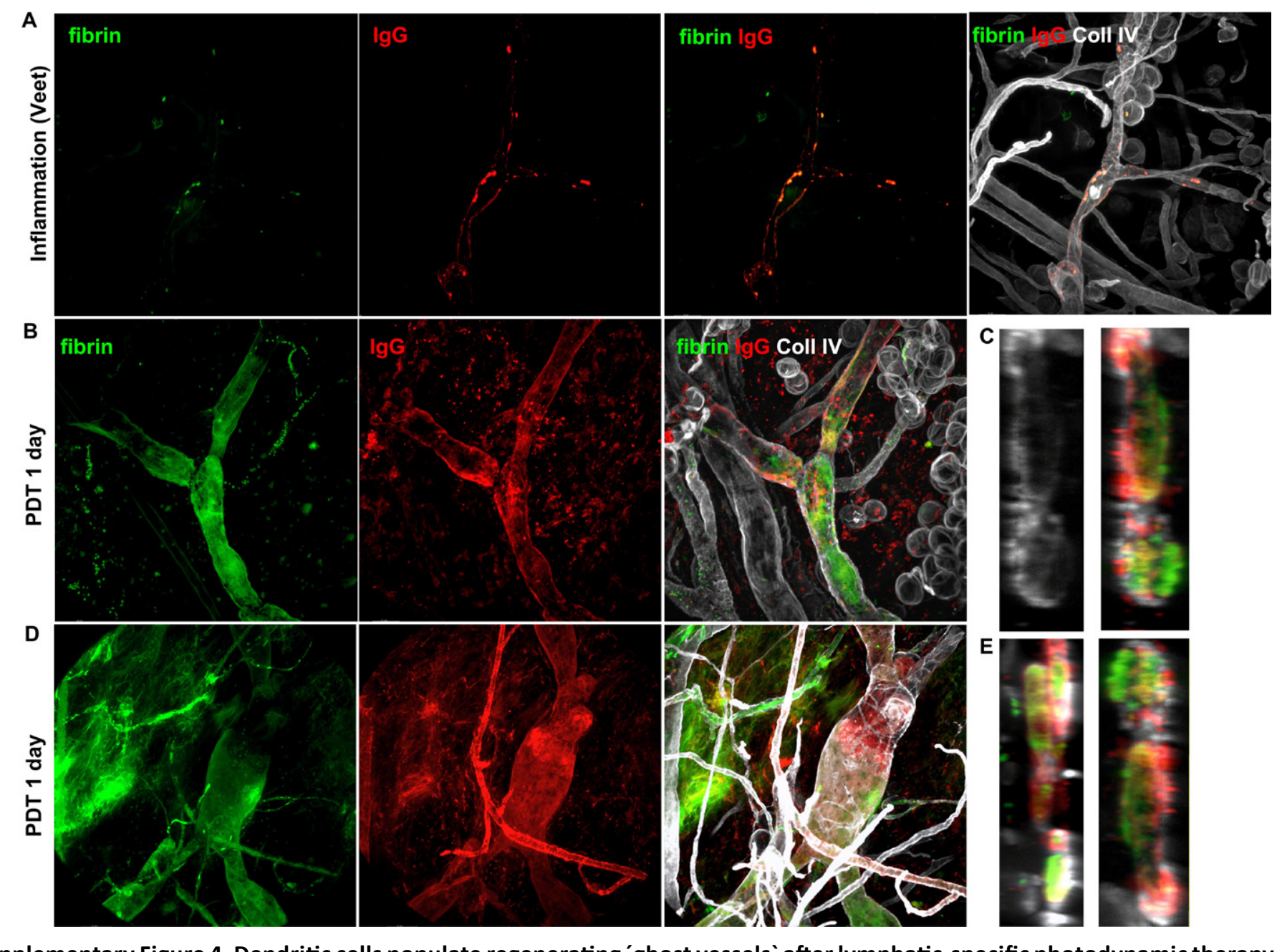

A



Day 3

Day 5

Day 7

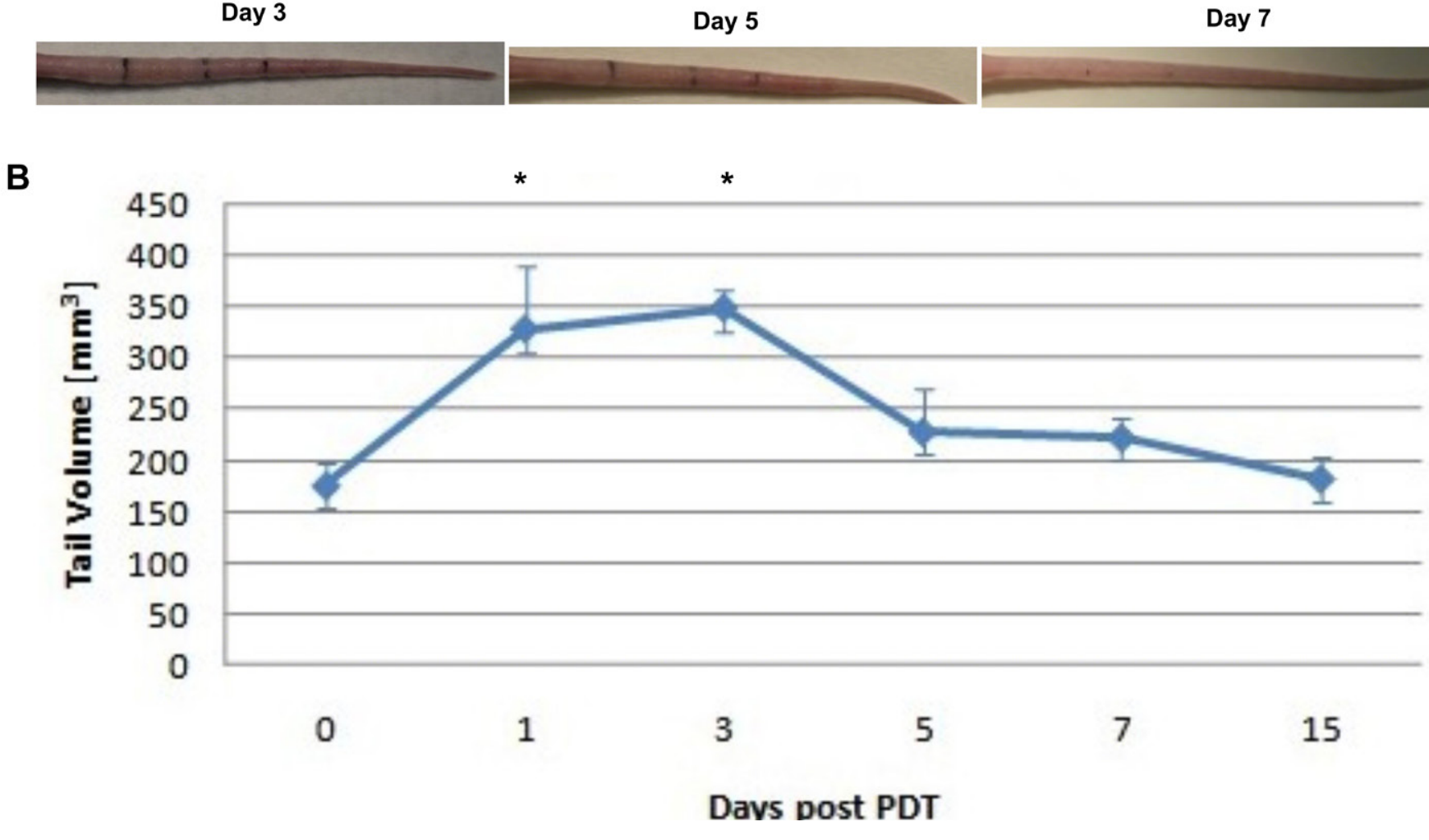

Supplementary Figure 5. Lymphatic occlusion alone does not lead to persistent edema

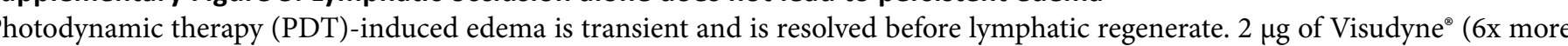
than used to occlude the ear lymphatics) was injected in two sides of the tail with FITC-dextran. Draining lymphatics at a distance of $4 \mathrm{~cm}$ of the tail were irradiated with $25 \mathrm{~J} / \mathrm{cm}^{2}$ and they were imaged with IVIS every day for 15 days. A. Example tail before edema development (day 0), during its peak (day 1 to 3 after PDT) and after the edema resolution (day 5, 7). B. Graph representing the
edema development as measured by tail volume, and resolution after PDT. 2-dimensional and dendrite interconnected network that stains strongly for MHCII and CD11c. Basement membrane (BM) is stained with collagen IV. B. In the control region of the ear treated with photodynamic therapy (PDT), lymphatics are not populated with antigen presenting cells. C. In contrast, lymphatic collectors decellularized with PDT are densely populated with mostly double- 